\title{
Scanning spacetime with patterns of entanglement
}

\author{
Péter Lévay $\odot$ and Bercel Boldis $(1)$ \\ MTA-BME Quantum Dynamics and Correlations Research Group, Department of Theoretical Physics, \\ Budapest University of Technology and Economics, 1521 Budapest, Hungary
}

(Received 31 January 2020; accepted 4 March 2020; published 24 March 2020)

\begin{abstract}
In the $\mathrm{AdS}_{3} / \mathrm{CFT}_{2}$ setup, we elucidate how gauge-invariant boundary patterns of entanglement of the CFT vacuum are encoded into the bulk via the coefficient dynamics of an $A_{N-3}, N \geq 4$, cluster algebra. In the static case, this dynamics of encoding manifests itself in kinematic space, which is a copy of de Sitter space $\mathrm{dS}_{2}$, in a particularly instructive manner. For a choice of partition of the boundary into $N$ regions, the patterns of entanglement, associated with conditional mutual information of overlapping regions, are related to triangulations of geodesic $N$-gons. Such triangulations are then mapped to causal patterns in kinematic space. For a fixed $N$, the space of all causal patterns is related to the associahedron $\mathcal{K}^{N-3}$, an object well known from previous studies on scattering amplitudes. On this space of causal patterns, cluster dynamics acts by a recursion provided by Zamolodchikov's $Y$ system of type $\left(A_{N-3}, A_{1}\right)$. We observe that the space of causal patterns is equipped with a partial order and is isomorphic to the Tamari lattice. The mutation of causal patterns can be encapsulated by a walk of $N-3$ particles interacting in a peculiar manner in the past light cone of a point of $\mathrm{dS}_{2}$.
\end{abstract}

DOI: 10.1103/PhysRevD.101.066021

\section{INTRODUCTION}

According to a recent exciting idea, entanglement patterns associated with certain boundary states of a conformal field theory (CFT) can manifest themselves in the classical geometry of the bulk [1-6]. This opens up the possibility of identifying spacetime structures as ones emerging holographically from entanglement data.

Recently, apart from the basic spaces featuring any holographic consideration, namely, the bulk and its boundary, another space called kinematic space has shown up. It is usually regarded as an intermediary between the bulk and boundary [7-11]. However, apart from translating between the language of quantum information of the boundary to the language of geometry of the bulk, kinematic space is also an interesting space in its own right. For example, in the $\mathrm{AdS}_{3} / \mathrm{CFT}_{2}$ setup, taking the static slice of $\mathrm{AdS}_{3}$ kinematic space $\mathbb{K}$ is just the space of geodesics for this static slice. It turns out that geometrically $\mathbb{K}$ is a copy of two-dimensional de Sitter space $\mathrm{dS}_{2}$. Then this picture has given rise to the idea of an emergent de Sitter from conformal field theory [9,12-16].

In our previous paper [17] using horocycles and their associated lambda lengths $[18,19]$, we have given a new geometric meaning to the well-known strong subadditivity

Published by the American Physical Society under the terms of the Creative Commons Attribution 4.0 International license. Further distribution of this work must maintain attribution to the author(s) and the published article's title, journal citation, and DOI. Funded by SCOAP ${ }^{3}$. relation $[20,21]$ for entanglement entropies. In particular, we related two aggregate measures on the boundary, detecting how far the infrared degrees of freedom are away from satisfying the strong subadditivity relation, to the shear coordinates of geodesic quadrangles in the bulk. This boundary measure, the conditional mutual information $[7,20]$ encapsulating the monogamy property of entanglement, is simply related to the geodesic distance between the geodesics forming the opposite sides of the quadrangle. Such geodesics have timelike separation as points in kinematic space. In the dual picture provided by kinematic space, the conditional mutual information is also related to the proper time along a timelike geodesic connecting the relevant two points of a causal diamond $[17,22]$.

This observation has revealed a connection with the theory of Teichmüller spaces of marked Riemann surfaces [18]. In particular, we have shown that the two different boundary measures of strong subadditivity are related to the two different triangulations of a geodesic quadrangle. In this picture, the shear coordinates [19], satisfying a reciprocal relation, are just possible local coordinates for the space of deformations of quadrangles, i.e., their Teichmüller space. Generalizing these observations for geodesic $N$-gons with $N \geq 4$, an interesting connection with $A_{N-3}$ cluster algebras [23-25] emerges. Here the cluster variables are just the lambda lengths [18] directly related to the regularized entropies of the boundary via the Ryu-Takayanagi relation [1].

We also emphasized that the basic role the gaugeinvariant conditional mutual information plays in these 
elaborations dates back to the unifying role of the Ptolemy relation for geodesic quadrangles [19]. This identity is the basis of recursion relations underlying transformation formulas for shear coordinates of geodesic polygons. In the general case of geodesic $N$-gons, these recursion relations are precisely of the form of Zamolodchikov's $Y$ system of $\left(A_{N-3}, A_{1}\right)$ type [26-29].

Moreover, we have also pointed out [17] that, since boundary intervals with their associated geodesics of the bulk are organized according to the causal structure of their corresponding points in kinematic space [7], cluster algebras are also connected to structures of causality in kinematic space. Finally, we have observed that the cluster dynamics based on flips (or, alternatively, on quiver mutations) provides a dynamics similar to the ones conjectured for holographic codes [30,31].

The aim of the present paper is to elaborate on these observations. In particular, following Ref. [17], we would like to give a detailed account of the correspondence between entanglement patterns of the boundary and their encoded images into the bulk and the kinematic space.

What we find is that the cluster dynamics provides different causal patterns consisting of causal diamonds in kinematic space which are related by cluster mutation. As is well known [7], the areas of the causal diamonds with respect to the Crofton form correspond to the conditional mutual information of the boundary. The novelty here is the result that these area labels for the causal diamonds are related to the coefficient variables of a cluster algebra in a simple manner. Moreover, we will see that the space of causal patterns is inherently related to the structure of the associahedron. One finds that the associahedron encapsulates the many different ways the kinematic space can be scanned by the periodic cluster dynamics. This dynamics is also encoded into the recursion relations of the corresponding Zamolodchikov $Y$ system. The simplest instance of this dynamics is the additive rule of conditional mutual information observed in Ref. [7]. One can conjecture that more intricate manifestations of this dynamics are probably related to some error-correction mechanism similar to the ones conjectured for holographic codes [30].

In this paper, for illustrative purposes we will be content with the simplest case based on the CFT vacuum, giving rise to cluster algebras of type $A_{N-3}$ with $N \geq 4$. More general scenarios are left to be explored in future work. Our hope is that these illustrations will shed some new light on issues concerning holographic codes [31] and their dynamics [30]. Moreover, they might also initiate further elaborations for exploring connections with a related research on scattering amplitudes which is also connected to the Arkani-Hamed-Bai-He-Yan amplituhedron [32] featuring similar geometric structures as the ones studied here.

The organization of this paper is as follows. In Sec. II, we review the basic background on the static slice of $\mathrm{AdS}_{3}$ which is represented by the Poincaré disk $\mathbb{D}$. The space of geodesics of $\mathbb{D}$ is kinematic space $\mathbb{K}$, which is a copy of de Sitter space $\mathrm{dS}_{2}$. In Sec. III, we summarize some of the results from Ref. [17]. Geodesic quadrangles and their associated lambda lengths, shears, and cross ratios appear here, quantities familiar from the theory of marked Riemann surfaces. The physical interpretation of these quantities is also given here. Next, in the spirit of Ref. [7] we relate areas in kinematic space to conditional mutual information, however, with a novel perspective provided by lambda lengths [18].

In Sec. IV, we present a detailed study on the algebraic structure of triangulations of geodesic polygons. Such triangulations in $\mathbb{D}$ are mapped to causal patterns in $\mathbb{K}$. Next, these causal patterns, made of causal diamonds, and their associated plaquette variables $X_{j, k}$, where $j=$ $1,2, \ldots N$ and $k=1,2, \ldots N-3$, are examined. They are related to conditional mutual information $I_{j, k}$ via Eq. (28). The plaquette variables satisfy a recursion relation (33), which after a change of variables takes the form of a Zamolodchikov $Y$ system (42). The solution of this system is well known [27] and can be reinterpreted in terms of conditional mutual information employing some seed variables. This result connects boundary entanglement structures to the coefficient dynamics [24] of a bulk recursive scanning process of $\mathbb{K}$ governed by an $A_{N-3}$ cluster algebra. In Sec. V, we show that apart from $X_{j, k}$ with $j+k \equiv 0 \bmod 2$ there are dual variables with $j+k \equiv 1$ mod 2. It turns out that the former set of variables is associated with points $(P)$, and the latter with complements of the light cones of such $P$ 's $(\bar{C})$ in $\mathrm{dS}_{2}$. Moreover, we demonstrate that an interesting relation [Eq. (52)] holds between the $P$ and $\bar{C}$ descriptions. Indeed, the difference between the corresponding mutual information is related to the geodesic length (proper time) between the timelike separated points in $\mathrm{dS}_{2}$ defining the relevant causal diamond labeled by $P$.

Cluster dynamics unfolds via operations called flips. In Sec. VI, we show that at the boundary level flips are represented by applying the semiassociative law of right or left shifts of binary bracketings introduced by Tamari [33]. This corresponds to a flip in the space of contexts of boundary regions represented by such binary bracketings. At the bulk level, they are represented by flips between the two possible diagonals of a geodesic quadrangle. This also corresponds to a flip in the space of triangulations of geodesic $\mathrm{N}$-gons. More importantly, at the kinematic space level a flip is represented by an elementary step taken by one of $N-3$ particles executing a random walk on the lattice inside a triangular region provided by the past light cone of a distinguished point. Here we also show that for fixed $N$ the space of causal patterns $T_{N-2}$ provides a set of regions covering $\mathrm{dS}_{2}$. The collection of such patterns of cardinality given by the Catalan number $C_{N-2}$ can be identified with the vertices of the associahedron $\mathcal{K}^{N-3}$. The boundaries of the associahedron of different dimensions 
correspond to different partial triangulations. In particular, the collection of facets of cardinality $N(N-3) / 2$ can be identified with the collection of Ryu-Takayanagi geodesics corresponding to diagonals. Finally, we establish on the space of causal patterns a partial order rendering this space a lattice $\left(T_{N-2}, \leq\right)$ isomorphic to the Tamari lattice. Our conclusions and an outlook relating our results to current research are left for Sec. VII.

\section{GEODESICS AND KINEMATIC SPACE}

$\mathrm{AdS}_{3}$ can be regarded as the hyperboloid in $\mathbb{R}^{2,2}$ characterized by the equation

$$
X^{2}+Y^{2}-U^{2}-V^{2}=-\ell_{\text {AdS }}^{2},
$$

where $\ell_{\text {AdS }}$ is the AdS radius. In the following, we fix $\ell_{\text {AdS }}=1$. In our considerations, we merely take the static (spacelike) slice of $\mathrm{AdS}_{3}$ by imposing $V=0$. We introduce coordinates for this slice as

$$
\begin{aligned}
& X=X_{1}=\sinh \varrho \cos \varphi, \\
& Y=X_{2}=\sinh \varrho \sin \varphi, \\
& U=X_{3}=\cosh \varrho,
\end{aligned}
$$

with $X^{2}+Y^{2}-U^{2}=-\ell_{\text {AdS }}^{2}$. Here $\varphi \in[0,2 \pi]$ and $\varrho \in[0, \infty)$. Then the set of points satisfying Eqs. (2) is the upper sheet $\mathbb{W}$ of the double-sheeted hyperboloid in $\mathbb{R}^{2,1}$ that can also be written as the coset space $\mathbb{U} \simeq S O(2,1) / S O(2)$.

After stereographic projection from the point $(X, Y, U)=(0,0,-1)$ of $\mathbb{t}$ to the Poincaré disk $\mathbb{D}$ lying in the plane $U=0$, we obtain the coordinates

$$
z=\tanh (\varrho / 2) e^{i \varphi}=\frac{X+i Y}{1+U}=x+i y \in \mathbb{D} .
$$

An alternative set of coordinates can be obtained by transforming to the upper half plane $\mathbb{U}$ by a Cayley transformation

$$
\tau=i \frac{1+z}{1-z}=\frac{i-Y}{U-X}=\xi+i \eta \in \mathbb{U}, \quad \eta>0 .
$$

In our static considerations, we will be referring to the spaces $\mathbb{D}$ and $\mathbb{U}$ as the bulk and the unit circle $S^{1} \simeq \partial \mathbb{D}$ and the compactified real line $\mathbb{R} \mathbb{P}^{1}=\mathbb{R} \cup\{\infty\} \simeq \partial \mathbb{U}$ as the boundary.

The metric $d s^{2}=d X^{2}+d Y^{2}-d U^{2}-d V^{2}$ on $\mathbb{R}^{2,2}$ and the choice of (2) coordinates for the $V=0$ slice yield the induced metric

$$
d s_{\boxplus}^{2}=d \varrho^{2}+\sinh ^{2} \varrho d \varphi^{2}
$$

on $\mathbb{H}$. Its geodesics are given by the formula

$$
\tanh \varrho \cos (\varphi-\theta)=\cos \alpha .
$$

They are circular arcs in the bulk starting and ending on the boundary; see Fig. 1 . Here the extra parameters $\theta \in[0,2 \pi]$ and $\alpha \in[0, \pi]$ are labeling the geodesics. Depicted on the disk $\mathbb{D}$, the coordinate $\theta$ is the center, and $\alpha$ is half the opening angle of the geodesic. Pairs of geodesics differing in orientation are related by $\theta \leftrightarrow \theta+\pi, \alpha \leftrightarrow \pi-\alpha$. Hence, our space of geodesics is labeled by the coordinates $(\alpha, \theta)$. It is called the kinematic space [7]. Topologically, the kinematic space $\mathbb{K}$ is the single-sheeted hyperboloid $S O(2,1) / S O(1,1)$, which is the de Sitter space $\mathrm{dS}_{2}$.

Note that one can alternatively use the coordinates $(u, v)$ related to the pair $(\alpha, \theta)$ as

$$
u=\theta-\alpha, \quad v=\theta+\alpha .
$$

The points $e^{i u}, e^{i v} \in \partial \mathbb{D}$ can then be regarded as the starting and the end points of a geodesic. If $\alpha$ is regarded as a timelike coordinate and $\theta$ as a spacelike one, then the pair of coordinates $(u, v)$ can be regarded as light cone coordinates.

Since kinematic space described by the coordinates $(\alpha, \theta)$ is a de Sitter space $\mathrm{dS}_{2}$ for a geodesic $\mathbf{R} \subset \mathbb{D}$ anchored to a boundary region $R \subset \partial \mathbb{D}$, one can associate a point $\mathcal{R} \in \mathbb{K}$ with three coordinates:

$$
\begin{aligned}
& \mathcal{R}_{1}=\frac{\cos \theta}{\sin \alpha}=\cosh \gamma \cos \theta, \\
& \mathcal{R}_{2}=\frac{\sin \theta}{\sin \alpha}=\cosh \gamma \sin \theta, \\
& \mathcal{R}_{3}=\cot \alpha=-\sinh \gamma,
\end{aligned}
$$

where the relation $\mathcal{R}_{1}^{2}+\mathcal{R}_{2}^{2}-\mathcal{R}_{3}^{2}=1$ holds and we introduced the coordinate transformation $\cosh \gamma=1 / \sin \alpha$.

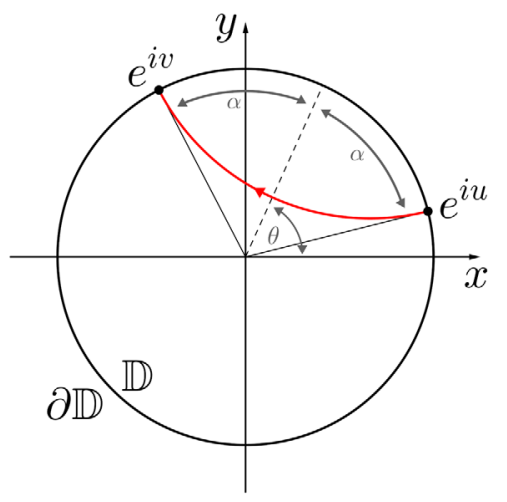

FIG. 1. The parametrization of a geodesic. The geodesic (red curve) is parametrized by the pair $(\theta, \alpha)$, where $\theta \in[0,2 \pi]$ and $\alpha \in[0, \pi]$. The coordinate $\theta$ is the center, and $\alpha$ is half the opening angle of the geodesic. Alternatively, one can use the pair $(u, v)$ defined by Eq. (7). 
Notice that $\mathbb{K}$ can be embedded in the same space $\mathbb{R}^{2,1}$ where $\mathbb{H}$ lives. Then by employing the coordinates of Eq. (8) the induced metric this time is of the form

$$
d s_{\mathbb{K}}^{2}=\frac{d \theta^{2}-d \alpha^{2}}{\sin ^{2} \alpha}=\frac{d u d v}{\sin ^{2} \frac{v-u}{2}} .
$$

Notice that by virtue of Eq. (6) our geodesics can alternatively be described by the constraint $X \cdot \mathcal{R}=0$; i.e., the vectors $X$ and $\mathcal{R}$ are Minkowski orthogonal, with the former a timelike and the latter a spacelike unit vector. Then the geodesics are described by the intersection of $\mathbb{M}$ with a plane in $\mathbb{R}^{2,1}$ with normal vector $\mathcal{R}$. The two different possible normal vectors give rise to the two points with coordinates $(\theta, \alpha)$ and $(\theta+\pi, \pi-\alpha)$ lying on $\mathbb{K}$, corresponding to the same geodesic curve with opposite orientations. As has been demonstrated in Ref. [34], the vector $\mathcal{R}$ can be also be regarded as the vector of conserved quantities for the geodesic motion on the pseudosphere $\mathbb{H}$.

Recall that according to Ref. [7] boundary intervals are organized according to causal structures formed by points located in kinematic space. These structures date back to the natural causal structure bulk geodesics enjoy based on the containment relation of boundary intervals. If two points in kinematic space are timelike separated, then their corresponding geodesics contain each other, have no intersection, and have the same orientation, and the corresponding boundary intervals are embedded. If they are null separated, then their geodesics have a common end point. Finally, if they are spacelike separated, then their corresponding geodesics either have an intersection or have different orientations without an intersection $[7,22]$.

\section{GEODESIC QUADRANGLES}

Let us fix four points $a, b, c$, and $d$ on the boundary $\partial \mathbb{D}$ arranged in a counterclockwise sense (see Fig. 2). These

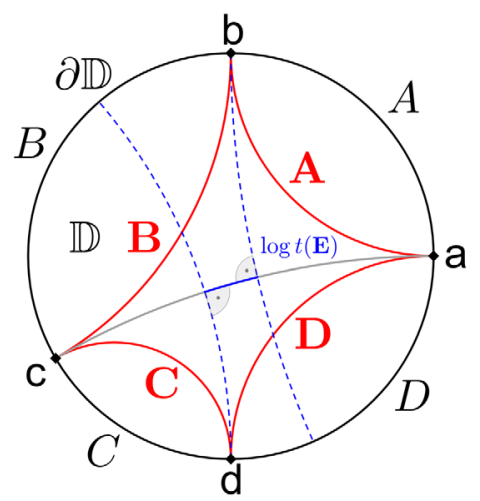

FIG. 2. A geodesic quadrangle, with the meaning of the shear coordinate $t(\mathbf{E})$ illustrated. The shear $\log t(\mathbf{E})$ of the geodesic quadrangle abcd is the hyperbolic length of that part of its diagonal $\mathbf{E}$, which lies in between the dashed geodesics intersecting $\mathbf{E}$ at a right angle. points give rise to four subregions: $A, B, C$, and $D$, where $A=[a, b], B=[b, c], C=[c, d]$, and $D=[d, a]$. Let us also consider the overlapping regions $E=[a, c]=A \cup B$ and $F=[b, d]=B \cup C$. Let us denote the oriented geodesics of the bulk $\mathbb{D}$ anchored to the corresponding boundary regions by $\mathbf{A}, \mathbf{B}, \mathbf{C}, \mathbf{D}, \mathbf{E}$, and $\mathbf{F}$. Consider also the corresponding points in kinematic space $\mathbb{K}$ denoted by $\mathcal{A}, \mathcal{B}, \mathcal{C}, \mathcal{D}, \mathcal{E}$, and $\mathcal{F}$. The complement of a boundary region $R$ will be denoted by $\bar{R}$, and both of these regions have the same geodesic arc anchored to them; however, $\mathbf{R}$ and $\overline{\mathbf{R}}$ have different orientations: counterclockwise for $\mathbf{R}$ and clockwise for $\overline{\mathbf{R}}$. The symbol $\mathcal{R}$ refers to the corresponding three-vector in $\mathbb{R}^{2,1}$ subject to the constraint $\mathcal{R}_{1}^{2}+\mathcal{R}_{2}^{2}-\mathcal{R}_{3}^{2}=1$, and $\overline{\mathcal{R}}$ refers to its negative.

According to strong subadditivity [20] for subregions $E$ and $F$ of the boundary for the von Neumann entanglement entropies, one has

$$
S(E)+S(F) \geq S(E \cup F)+S(E \cap F) .
$$

Note that $\bar{D}=A \cup B \cup C=E \cup F, E \cap F=B$. Denoting the union of boundary regions by juxtaposition, one can also write

$$
S(A B)+S(B C) \geq S(B)+S(A B C) .
$$

For pure states, one has $S(\bar{D})=S(D)$ and $S(B C)=S(A D)$; hence,

$$
S(A B)+S(A D) \geq S(B)+S(D) .
$$

Notice that, unlike in the classical (Shannon) case, in the quantum case it is possible to have either $S(B)>S(A B)$ or $S(D)>S(A D)$. In spite of this, the sum of these two inequalities can conspire in a manner such that Eq. (12) still holds.

One can also express the measures of strong subadditivity in terms of the conditional mutual information as

$I(A, C \mid B)=S(A B)+S(B C)-S(B)-S(A B C) \geq 0$,

where

$$
I(A, C \mid B) \equiv S(A \mid B)-S(A \mid B C) \geq 0
$$

with $S(A \mid B)=S(A B)-S(B)$ the conditional entropy. The quantities on the left-hand sides indicate that conditioning on a larger subsystem can only reduce the uncertainty about a system. Alternatively, Eq. (12) can be rephrased as

$$
S(A \mid B)+S(A \mid D) \geq 0,
$$

meaning that subsystem $A$ can be entangled with $B$ reducing $S(A B)$, or with $D$ reducing $S(A D)$, but not both. 
This fact is related to the notion of the monogamy of entanglement.

In this paper, we would like to obtain new insight into the issue of how these boundary data of quantum information are encoded into bulk data of classical geodesic geometry. As the first step in achieving this task, we recall that data on conditional mutual information can be expressed in terms of cross ratios $[7,21]$. In order to see this and also to give to this connection a new meaning needed later, we proceed as follows.

First, we notice that in terms of either coordinates on $\partial \mathbb{U}$ or on $\partial \mathbb{D}$ we have

$$
[d, b ; a, c] \equiv \frac{\left(\xi_{d}-\xi_{a}\right)\left(\xi_{b}-\xi_{c}\right)}{\left(\xi_{d}-\xi_{c}\right)\left(\xi_{b}-\xi_{a}\right)}=\frac{\sin \left(\frac{\varphi_{d}-\varphi_{a}}{2}\right) \sin \left(\frac{\varphi_{b}-\varphi_{c}}{2}\right)}{\sin \left(\frac{\varphi_{d}-\varphi_{c}}{2}\right) \sin \left(\frac{\varphi_{b}-\varphi_{a}}{2}\right)}
$$

where $[d, b ; a, c]$ is the cross ratio on $\mathbb{R P}^{1}$. Let us then denote the negative of this cross ratio by $t(\mathbf{E})$ and for a boundary subregion $R$ introduce the lambda length [18] $\lambda(\mathbf{R})$ of the corresponding geodesic $\mathbf{R}$. Then in terms of these data one gets the alternative expression [17]

$$
t(\mathbf{E})=-[d, b ; a, c]=\frac{\lambda(\mathbf{B}) \lambda(\mathbf{D})}{\lambda(\mathbf{A}) \lambda(\mathbf{C})} .
$$

Note that the lambda length (see Fig. 3) is related to the regularized geodesic length introduced in Ref. [35] and, hence, via the Ryu-Takayanagi proposal [1] to the entanglement entropy of the corresponding boundary interval. For a detailed discussion on this relationship, see Appendix A in Ref. [17].

The calculation of entanglement entropy for boundary regions is cutoff dependent. However, the boundary description of the "space of cutoffs" can be transcribed to the bulk description to the "space of horocycles," which is a homogeneous space $\mathbb{G}$. In Ref. [17], we have argued

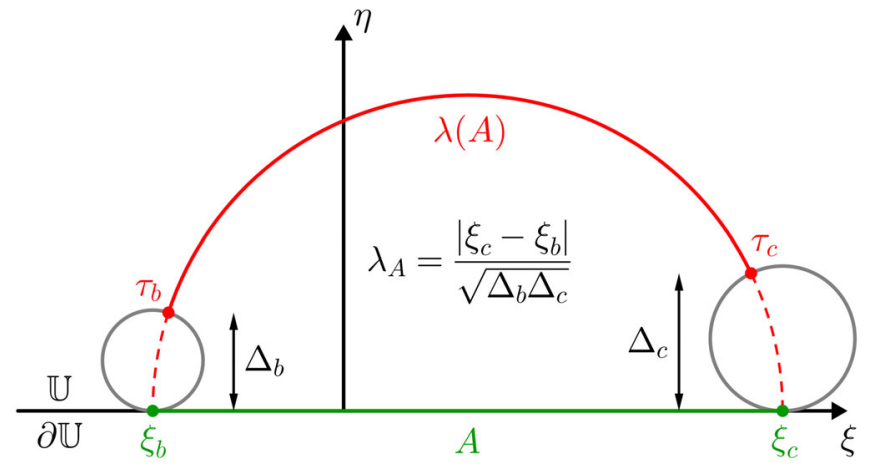

FIG. 3. Illustration of the meaning of the lambda length for a circular arc centered on $\mathbb{R}$ which is a part of the boundary $\partial \mathbb{U}$. According to Penner [18], the lambda length is the regularized length of the geodesic, where the regulators are horocycles. On $\mathbb{U}$, these are circles with Euclidean diameters $\Delta$, providing a natural cutoff. that it is worth regarding the choice of horocycles as a gauge-fixing procedure of the gauge degree of freedom noticed in Ref. [10]. In this picture, the geometrization of the cutoff dependence of the entanglement entropy corresponds to the horocycle dependence of the lambda length. In light of this, physical quantities independent of the choice of horocycles (cutoffs) will be called gaugeinvariant ones.

Now according to these results $t(\mathbf{E})$ has the dual interpretation as the ratio of Euclidean lengths $\frac{B D}{A C}$ in the boundary $\partial \mathbb{U}$ of the upper half plane and also as the gaugeinvariant ratio of lambda lengths $\frac{\lambda(\mathbf{B}) \lambda(\mathbf{D})}{\lambda(\mathbf{A}) \lambda(\mathbf{C})}$ in the bulk $\mathbb{U}$. More importantly, $\log t(\mathbf{E})$ can also be regarded as a gaugeinvariant shear coordinate [17] characterizing the deformation of geodesic quadrangles. Alternatively, one can regard $\log t(\mathbf{E})$ as a coordinate for the Teichmüller space of $\mathbb{D}$ with four marked points $[18,19]$. For an illustration of the meaning of the shear coordinate, see Fig. 2.

The geodesics $\mathbf{E}$ and $\mathbf{F}$ are the diagonals of the geodesic quadrangle ABCD. One can define a shear coordinate for both such diagonals. It is easy to show that

$$
t(\mathbf{F})=-[b, d ; a, c]=\frac{\lambda(\mathbf{A}) \lambda(\mathbf{C})}{\lambda(\mathbf{B}) \lambda(\mathbf{D})} .
$$

This means that $t(\mathbf{E}) t(\mathbf{F})=1$ and these shears serve as alternative local coordinates for the Teichmüller space of bulk geodesic quadrangles.

The area form associated to the metric of Eq. (9) is related to the Crofton form $\omega$ on kinematic space. More precisely, one has [7]

$$
\omega=\frac{\partial^{2} S(u, v)}{\partial u \partial v} d u \wedge d v=\frac{\mathfrak{c}}{12} \frac{d u \wedge d v}{\sin ^{2}\left(\frac{v-u}{2}\right)},
$$

where

$$
S(u, v)=\frac{\mathfrak{c}}{3} \log \left(e^{\Lambda} \sin \left(\frac{v-u}{2}\right)\right)
$$

with $e^{\Lambda}$ the cutoff factor. Here $\mathfrak{c}$ is the central charge of the boundary CFT related to the bulk Newton constant $G_{\mathrm{N}}$ and the AdS length scale $\ell_{\text {AdS }}$ via the Brown-Henneaux [36] relation: $\mathfrak{c}=\frac{3 \ell_{\text {Ads }}}{2 G_{\mathrm{N}}}$.

Calculating the integral

$$
\int_{\varphi_{a}}^{\varphi_{b}} \int_{\varphi_{c}}^{\varphi_{d}} \frac{d u \wedge d v}{4 \sin ^{2}\left(\frac{v-u}{2}\right)}=\log (1+t(\mathbf{F})),
$$

one proves that the areas calculated with the Crofton form are just the conditional mutual information; moreover, in terms of these shear coordinates, one has [17] 


$$
\begin{aligned}
& I(A, C \mid B)=\frac{\mathfrak{c}}{3} \log (1+t(\mathbf{F})) \geq 0 \\
& I(B, D \mid A)=\frac{\mathfrak{c}}{3} \log (1+t(\mathbf{E})) \geq 0 .
\end{aligned}
$$

As a consequence of $t(\mathbf{E}) t(\mathbf{F})=1$, one also has

$$
I(A, C \mid B)-I(B, D \mid A)=\frac{\mathfrak{c}}{3} \log t(\mathbf{F}) .
$$

This equation gives the kinematic space interpretation of the shear $\log t(\mathbf{F})$ in terms of the difference in areas of the causal diamonds encoding the corresponding conditional mutual information.

A further geometric interpretation to conditional mutual information can be given by noticing that [19]

$$
1+t(F)=\cosh ^{2} \frac{\ell}{2}, \quad 1+t(E)=\cosh ^{2} \frac{\ell^{\prime}}{2},
$$

where $\ell\left(\ell^{\prime}\right)$ is the infimum of the geodesic distances between the geodesics $\mathbf{B}$ and $\mathbf{D}$ (A and $\mathbf{C}$ ). Let us then denote by $(\overline{\mathcal{A}}, \ldots, \overline{\mathcal{F}})$ the negatives of the corresponding vectors representing oppositely oriented geodesics in $\mathbb{K}$. Then using (22) we have

$$
\cosh \ell=\overline{\mathcal{B}} \cdot \mathcal{D}, \quad \cosh \ell^{\prime}=\overline{\mathcal{A}} \cdot \mathcal{C}
$$

expressing the infimum of the geodesic distances between the pairs of geodesics $(\overline{\mathbf{B}}, \mathbf{D})$ and $(\overline{\mathbf{A}}, \mathbf{C})$ in $\mathbb{D}$ in terms of the data of the corresponding pair of points $(\overline{\mathcal{B}}, \mathcal{D})$ and $(\overline{\mathcal{A}}, \mathcal{C})$ in $\mathbb{K}$. Notice that using the metric of Eq. (9) in $\mathbb{K}$ one can show [22] that the proper time $\Delta \tau$ between the two points $\mathcal{D}$ and $\overline{\mathcal{B}}$ along a timelike geodesic is just the geodesic length $\ell$ between the geodesics $\mathbf{D}$ and $\overline{\mathbf{B}}$ of $\mathbb{D}$; i.e., we have $\Delta \tau=\ell$. Likewise, the proper time $\Delta \tau^{\prime}$ between the two points $\mathcal{C}$ and $\overline{\mathcal{A}}$ along a timelike geodesic is just the geodesic length $\ell^{\prime}$ between the geodesics $\mathbf{C}$ and $\overline{\mathbf{A}}$ of $\mathbb{D}$; i.e., we have $\Delta \tau^{\prime}=\ell^{\prime}$. Combining these results with the previous ones, we obtain the formulas

$$
\begin{aligned}
& I(A, C \mid B)=\frac{\ell_{\mathrm{AdS}}}{G_{\mathrm{N}}} \cdot \log \cosh \frac{\ell}{2}, \\
& I(B, D \mid A)=\frac{\ell_{\mathrm{AdS}}}{G_{\mathrm{N}}} \cdot \log \cosh \frac{\ell^{\prime}}{2} .
\end{aligned}
$$

These results show that gauge-invariant boundary measures of entanglement can be expressed in terms of geometric data of either the bulk $(\ell)$ or kinematic space $(\Delta \tau)$; see Fig. 4.

\section{THE ALGEBRAIC STRUCTURE OF GEODESIC POLYGONS IN KINEMATIC SPACE}

For geodesic $N$-gons, we label the $N$ points on the boundary $\partial \mathbb{D}$ by the numbers $\{0,1,2, \ldots N-1\}$. By the use of $n=N-3$ diagonals, one can obtain a triangulation for these $N$-gons. The $N$ boundary points give rise to point curves [7] in $\mathbb{K}$. They are just trajectories of light rays. The set of $N$ left-moving and $N$ right-moving light rays forms a grid for $\mathbb{K}$. Our basic idea is to study the algebra of the area labels of causal diamonds, i.e., of the rectangular regions bounded by four different point curves. We know that the areas of such regions encode conditional mutual information. We would like to obtain an algebraic characterization of these labeled regions encoding entanglement patterns of the CFT vacuum.

Similar to the one of Fig. 4, now in Fig. 5 we see a grid of point curves for $N=4$. However, now we switched to the new labeling $\{a, b, c, d\} \leftrightarrow\{0,1,2,3\}$. A quadruplet of

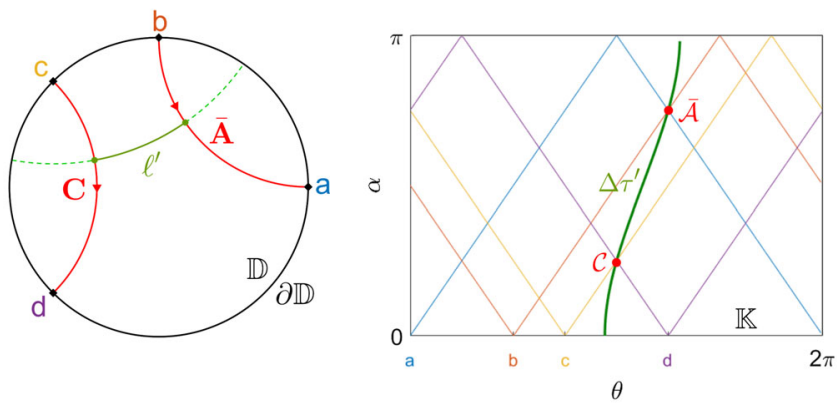

FIG. 4. The geodesic length between geodesics in $\mathbb{D}$ corresponds to proper time in $\mathbb{K}$ between timelike separated points. Note that according to Eq. (6) the colored points on $\partial \mathbb{D}$ correspond to colored point curves (light rays) in $\mathbb{K}$.
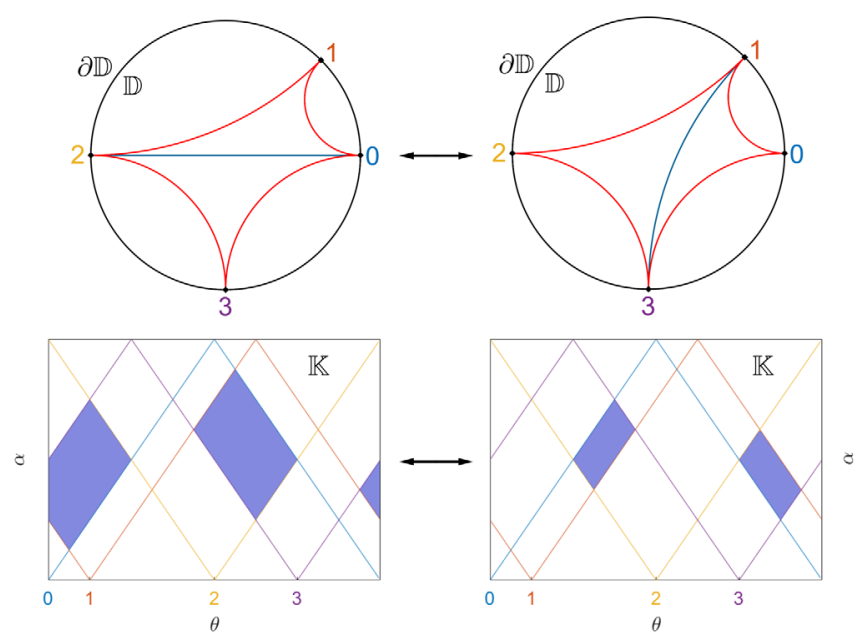

FIG. 5. The triangulations of a geodesic quadrangle related by a flip in the bulk and the associated causal patterns in kinematic space. 
boundary points forms a quadrangle in the bulk. Choosing a particular diagonal from the two possible ones of this quadrangle gives rise to a shear coordinate. For example, for the quadrangle labeled by the set $\{0,1,2,3\}$, we define $u \equiv t_{12,30}=t_{30,12}$ as

$$
t_{12,30}=-[1,3 ; 2,0]=-\frac{\sin \left(\frac{\varphi_{1}-\varphi_{2}}{2}\right) \sin \left(\frac{\varphi_{3}-\varphi_{0}}{2}\right)}{\sin \left(\frac{\varphi_{1}-\varphi_{0}}{2}\right) \sin \left(\frac{\varphi_{3}-\varphi_{2}}{2}\right)} .
$$

The quantity $u$ is associated to the diagonal connecting the points 0 and 2. Clearly, for the diagonal connecting the ones 1 and 3 , we can define $t_{01,23}=t_{23,01}=1 / u$. In this notation, we have

$$
\begin{aligned}
& I(12,30 \mid 23)=\frac{\mathfrak{c}}{3} \log \left(1+t_{12,30}\right)=\frac{\mathfrak{c}}{3} \log (1+u), \\
& I(01,23 \mid 12)=\frac{\mathfrak{c}}{3} \log \left(1+t_{01,23}\right)=\frac{\mathfrak{c}}{3} \log \left(1+\frac{1}{u}\right),
\end{aligned}
$$

where entries like 12 indicate the corresponding boundary region, i.e., the one between the points 1 and 2 . Notice that for a cyclic (counterclockwise) orientation of $\{0,1,2,3\}$ the $t$ 's are positive. According to Fig. 2, their logarithms describe shears.

Now for a geodesic $N$-gon with a choice for its $n=$ $N-3$ diagonals (giving rise to a triangulation), we define for $j \in \mathbb{Z}$ and $k=1,2, \ldots, n$ a special set of variables:

$$
\frac{1}{X_{j, k}}=1+t_{a b, c d}, \quad j+k \equiv 0 \quad \bmod 2
$$

where

$$
\begin{aligned}
& b \equiv \frac{j-k}{2}, \quad c \equiv \frac{j+k}{2}, \quad a \equiv b-1, \\
& d \equiv c+1 \quad \bmod (n+3) .
\end{aligned}
$$

This special set of variables defines a special set of conditional mutual information:

$$
I_{j, k} \equiv I(a b, c d \mid b c), \quad j \in \mathbb{Z}, \quad k=1, \ldots, n,
$$

where Eqs. (26a) and (26b) show that

$$
X_{j, k}=e^{-(3 / \mathfrak{c}) I_{j, k}}, \quad j+k \equiv 0 \quad \bmod 2 .
$$

For example, for a geodesic pentagon $N=5, n=2$ with a cyclic labeling $\{0,1,2,3,4\}$, we choose the triangulation by considering the diagonals 03 and 02 . They feature the geodesic quadrangles 2340 and 1230. We associate to them the positive quantities $u_{1}=t_{40,23}=t_{23,40}$ and $u_{2}=t_{30,12}=t_{12,30}$. Then with this choice of triangulation and values for the pair $\left(u_{1}, u_{2}\right)$ we have

$$
\begin{aligned}
\frac{1}{X_{1,1}} & \equiv 1+\frac{u_{1} u_{2}}{1+u_{1}}=1+t_{40,12}, \\
\frac{1}{X_{2,2}} & \equiv 1+u_{1}=1+t_{40,23}, \\
\frac{1}{X_{3,1}} & \equiv 1+\frac{1}{u_{2}}=1+t_{01,23}, \\
\frac{1}{X_{4,2}} & \equiv 1+\frac{1}{u_{1}\left(1+u_{2}\right)}=1+t_{01,34}, \\
\frac{1}{X_{5,1}} & \equiv 1+\frac{u_{2}}{1+u_{1}+u_{1} u_{2}}=1+t_{12,34 .}
\end{aligned}
$$

Notice that we can extend this set by adjoining to them new plaquette variables satisfying the formula

$$
X_{j+5,3-k}=X_{j, k}, \quad j=0,1,2,3,4 .
$$

Now, according to Eq. (19), the logarithms of these quantities are proportional to the areas of the regions in $\mathbb{K}$ that are bounded by point curves labeled by the numbers $a, b, c, d \in\{0,1, \ldots N-1\}$ showing up in $t_{a b, c d}$. More precisely, according to Eq. (17), $\frac{c}{3}$ times these logarithms give the areas of such regions with respect to the Crofton form. These areas in kinematic space encode conditional mutual information of the corresponding regions in $\partial \mathbb{D}$. Notice that, due to the periodicity of kinematic space, by also using the adjoint set of variables of Eq. (30) no more $X_{j, k}$ plaquette variables are needed. Indeed, the central belt of $\mathbb{K}$ is already covered by them. This is reflected in the periodicity property $X_{j+10, k}=X_{j, k}$.

As an example, one can consider the pair of variables $\left(X_{5,1}, X_{6,2}\right)=\left(X_{5,1}, X_{1,1}\right)$. According to Figs. 6 and 7 , taken together the corresponding two regions cover the region between the point curves of the boundary points 1,2 and 3,0. Taking the logarithm of $\left(X_{5,1} X_{6,2}\right)^{-1}$, which is
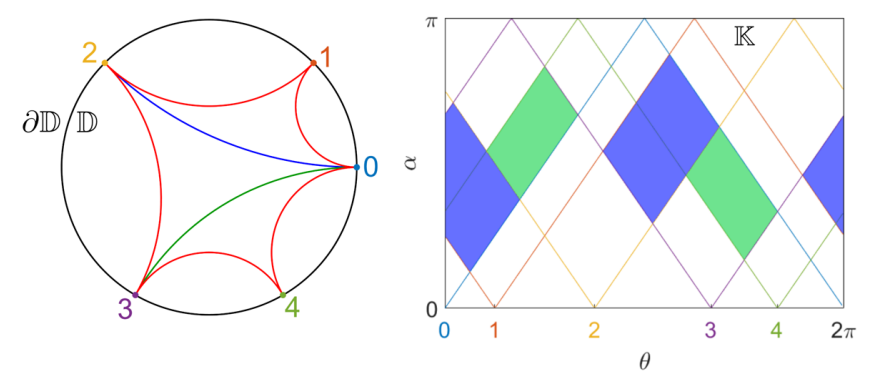

FIG. 6. A triangulation of a geodesic pentagon and the associated causal pattern in kinematic space. The causal pattern is the one answering the boundary binary bracketing of regions of the form $\left(\left(\left(R_{0} R_{1}\right) R_{2}\right) R_{3}\right)$. See Sec. VI for notation. 


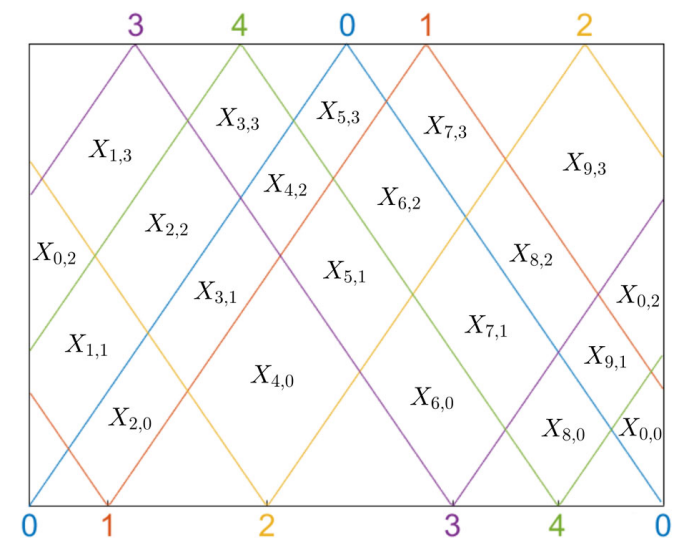

FIG. 7. Cluster labeling of the kinematic space for a geodesic pentagon in Fig. 6.

$$
\left(1+\frac{u_{2}}{1+u_{1}+u_{1} u_{2}}\right)\left(1+\frac{u_{1} u_{2}}{1+u_{1}}\right)=1+u_{2}
$$

and recalling that $u_{2}=t_{12,30}$ illustrates the additivity of conditional mutual information [7], i.e., $I(12,34 \mid 23)+$ $I(12,40 \mid 24)=I(12,30 \mid 23)$. This reproduces a well-known algebraic property of these measures of entanglement.

However, one can also reveal a more intricate algebraic structure of these measures. In order to elaborate on this structure, it is worth introducing new variables $X_{j, 0}$ and $X_{j, n+1}$. They will correspond to regions extending to the conformal boundary of $\mathbb{K}$. Indeed, geodesics with a zero opening angle correspond to points on $\partial \mathbb{D}$, and such geodesics are located at the conformal boundary of kinematic space [14]. Now unlike the $X_{j, k}$ 's with $k=1,2, \ldots n$ encoding conditional mutual information like $I(A, C \mid B)$, the new variables are encoding mutual information of the form $I(A, B)=S(A)-S(A B)+S(B)$. However, since these regions are extending to the conformal boundary of $\mathbb{K}$, they have diverging areas with respect to the Crofton form. Notice that this is as it should be, since, unlike the $I(A, C \mid B)$ 's, the $I(A, B)$ 's are cutoff dependent (divergent) quantities of $\partial \mathbb{D}$. The resulting divergence can explicitly be seen, for example, by taking the limit $\varphi_{3} \rightarrow \varphi_{2}$ in Eq. (25), yielding a diverging $t_{12,30}$. Now due to Eq. (27) such limiting cases yield vanishing values for these new variables: $X_{j, 0}=X_{j, n+1}=0$. In the following, we implement these constraints as boundary conditions for the variables $X_{j, k}$.

Now the important observation we would like to make is that for the (28) set of conditional mutual information under the boundary conditions

$$
X_{j, 0}=X_{j, n+1}=0
$$

the following recursion relation holds:

$$
\begin{aligned}
& \left(1-\frac{1}{X_{j+1, k}}\right)\left(1-\frac{1}{X_{j-1, k}}\right) \\
& \quad=\left(1-X_{j, k+1}\right)\left(1-X_{j, k-1}\right) .
\end{aligned}
$$

It is well known that one can explicitly solve this recursion relation [27]. The solution rests on choosing a special set of $X_{j, k}$ 's which we call a seed set. In the notation of Ref. [27], let us define $a_{k} \equiv X_{k, k}$, where $k=1, \ldots, n$ with the boundary condition of Eq. (32) for $j=0$ and $j=$ $n+1$ translated into $a_{0}=a_{n+1}=0$. Then

$$
\begin{aligned}
X_{j+2, j} & =\frac{1-a_{1} a_{2} \ldots a_{j}}{1-a_{1} a_{2} \ldots a_{j+1}}, \\
X_{n-j-1, n-j+1} & =\frac{1-a_{n} a_{n-1} \ldots a_{n+1-j}}{1-a_{n} a_{n-1} \ldots a_{n-j}} .
\end{aligned}
$$

Using this solution, one can prove that the solution of the (33) system is periodic in $j \in \mathbb{Z}$; i.e., one can prove that

$$
X_{j+n+3, n+1-k}=X_{j, k}
$$

[see also Eq. (30) for the $n=2$ case], which yields $X_{j+2 N, k}=X_{j, k}$ with $N=n+3$.

Notice that for geodesic $N$-gons the number of nonzero seed variables equals $n$, which is the number of diagonals of a triangulations. Hence, choosing a particular triangulation of a geodesic $\mathrm{N}$-gon, one can derive a set of nonzero seed values $a_{k}, k=1,2, \ldots n$. Using then the explicit solution of Eq. (33), one can generate the conditional mutual information labels for all of the causal diamonds covering the central belt of $\mathbb{K}$. In this way, for a particular triangulation one obtains a labeled tiling of kinematic space by patterns of entanglement of the CFT vacuum. For example, for the $N=5, n=2$ case after choosing the triangulation shown in Fig. 6 with the associated shear coordinates $\left(u_{1}, u_{2}\right)$, the seed variables are $X_{1,1}$ and $X_{2,2}$ of Eqs. (29a) and (29b). These are the plaquette variables for the leftmost side of the central belt shown in Fig. 7. Then using Eq. (34) and (35) and the periodicity property, one can generate all the variables for the central belt.

As another example, one can take the $N=6, n=3$ case of the regular hexagon with the chosen triangulation given by the one in Fig. 8. The shear coordinates associated to the corresponding diagonals are

$$
\left(u_{1}, u_{2}, u_{3}\right)=\left(t_{02,34}, t_{12,40}, t_{24,50}\right)
$$

These coordinates give rise to the seed variables 

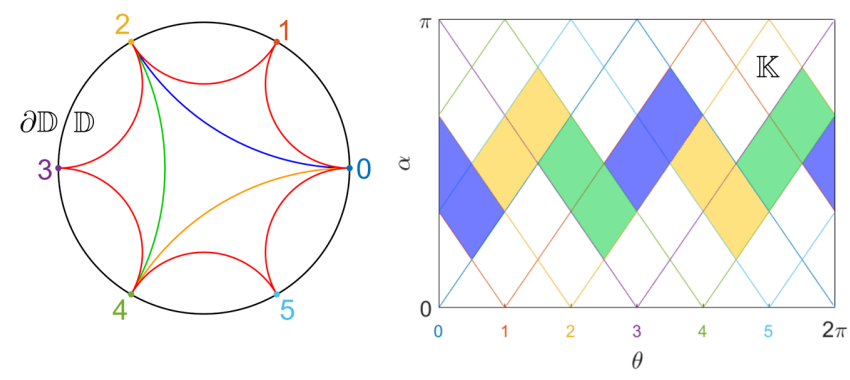

FIG. 8. A triangulation of a regular geodesic hexagon and the associated causal pattern in kinematic space. The causal pattern is the one answering the boundary binary bracketing of regions of the form $\left(\left(\left(R_{0} R_{1}\right)\left(R_{2} R_{3}\right)\right) R_{4}\right)$. For notation, see Sec. VI.

$$
\begin{aligned}
& a_{1}=X_{1,1}=\frac{1+u_{2}}{1+u_{2}+u_{2} u_{3}}, \\
& a_{2}=X_{2,2}=\frac{1+u_{1}+u_{1} u_{2}}{\left(1+u_{1}\right)\left(1+u_{2}\right)}, \\
& a_{3}=X_{3,3}=\frac{1+u_{1}}{1+u_{1}+u_{1} u_{2}} .
\end{aligned}
$$

In this case, the seed corresponds to the three causal wedges in between the two blue lines in the leftmost part of the central belt in Fig. 9. From this seed by a straightforward exercise one can generate all the conditional mutual information labels for the remaining causal diamonds.

It is important to realize that for regular $\mathrm{N}$-gons (see Fig. 9 for the $N=6$ case) the conditional mutual information $I_{j, k}$ depends only on $k=1,2, \ldots, n$. Indeed, since the (9) metric on kinematic space depends merely on $\alpha$, the

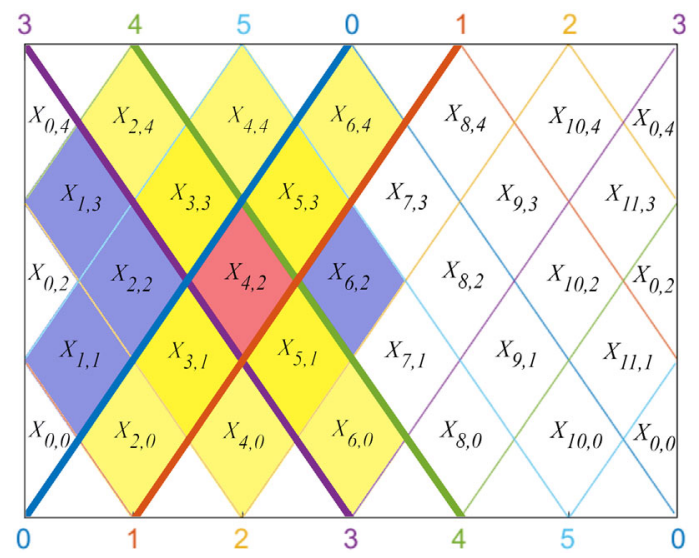

FIG. 9. Cluster labeling of kinematic space for a regular geodesic hexagon. The light cone of the variable $X_{4,2}$ highlighted in red is shown. The causal diamonds inside and on its light cone are the yellow regions, and the diamonds outside are the blue ones. Because of Zamolodchikov periodicity, the causal diamonds belonging to a fundamental domain are colored with blue and dark yellow. Notice that merely the blue diamonds $\left(\overline{C_{4,2}}\right)$ and the red one of the fundamental domain belonging to the central belt of $\mathbb{K}$ feature Eq. (50). areas of the causal diamonds in the regular $N$-gon case are depending merely on the discretized value of $\alpha$, which is the label $k$. One can also see this from the explicit form of the (25) shear coordinate, which (in an obvious notation) gives

$$
t_{j, k}=\frac{\sin ^{2} \kappa}{\sin ((k+2) \kappa) \sin (k \kappa)}, \quad \kappa=\frac{\pi}{N},
$$

a quantity independent of $j$, which is the discretization of the coordinate $\theta$. This result gives the explicit form of the $j$-independent conditional mutual information for regular $N$-gons:

$I_{j, k}=\frac{\mathfrak{c}}{3} \log \frac{\sin ^{2}((k+1) \kappa)}{\sin ^{2}((k+1) \kappa)-\sin ^{2} \kappa}, \quad k=1, \ldots, n$.

Employing now the basic relation of Eq. (28), one obtains for the $j$-independent plaquette variables

$$
X_{j, k}^{(0)} \equiv 1-\frac{\sin ^{2} \kappa}{\sin ^{2}((k+1) \kappa)},
$$

familiar from Ref. [27].

Let us finally connect our patterns of entanglement to some structures well known in the literature. First of all, notice that the $j$-independent expression of Eq. (40) solves our basic equation of Eq. (33). The explicit form of the $j$-independent version of this equation can be written in the form

$$
X_{j, k}^{2}=\prod_{l=1}^{n}\left(1-X_{j, k}\right)^{A_{k l}},
$$

where $A_{k l}$ is the Cartan matrix of the Dynkin diagram of type $A_{n}$.

Generally, let us define

$$
Y_{j, k}=\frac{X_{j, k}}{1-X_{j, k}}=\frac{1}{e^{(3 / \mathfrak{c}) I_{j, k}}-1},
$$

where $j \in \mathbb{Z}$ and $k=1,2, \ldots, n$. Then the new form of the (33) recursion relation is

$$
Y_{j-1, k} Y_{j+1, k}=\left(1+Y_{j, k-1}\right)\left(1+Y_{j, k+1}\right)
$$

with boundary conditions $Y_{j, 0}=Y_{j, n+1}=0$. This recursion is defining a Zamolodchikov $Y$ system of type $\left(A_{n}, A_{1}\right)$ [26]. It is known that any solution of the thermodynamic Bethe ansatz equations for a certain type of theories labeled by pairs $(G, H)$ of Dynkin diagrams of $A D E$ type satisfies a set of equations which boils down to a recursion generalizing the (43) form [28]. 
The result $[27,29]$

$$
Y_{i+n+3, n+1-j}=Y_{i, j}
$$

is called Zamolodchikov periodicity. In our treatise, it shows up naturally in the scanning process of 2D de Sitter spacetime (now playing the role as the kinematic space $\mathbb{K}$ ) by entanglement patterns. In closing this section, let us summarize the basic relations between the plaquette variables $X_{j, k}$ and $Y_{j, k}$ and the shear coordinate $t_{j, k}$ :

$$
\frac{1}{X_{j, k}}=1+\frac{1}{Y_{j, k}}=1+t_{j, k} .
$$

\section{DUALITY}

We have seen that our encoding of entanglement patterns into kinematic space was based on a Zamolodchikov $Y$ system of type $\left(A_{n}, A_{1}\right)$. The structure of $Y$ systems of type $(G, H)$ is determined by the adjacency structure of the corresponding Dynkin diagrams. Now the set of vertices $\mathcal{I}$ of any Dynkin diagram is a disjoint union of two sets $\mathcal{I}_{+}$ and $\mathcal{I}_{-}$such that there is no edge between any two vertices of $\mathcal{I}_{+}$nor between any two vertices of $\mathcal{I}_{-}$. For the Dynkin diagram of type $A_{n}$, this means that for $\mathcal{I} \simeq\{1,2, \ldots, n\}$ we have two types of vertices labeled by even or odd numbers. Let us then define for $k=1,2, \ldots, n$

$$
\epsilon(k)= \begin{cases}+1, & \text { if } k \text { is even, } \\ -1, & \text { if } k \text { is odd }\end{cases}
$$

and let

$$
b_{k l}=2 \delta_{k l}-A_{k l}
$$

be the adjacency matrix of the Dynkin diagram of type $A_{n}$, where $A_{k l}$ is the corresponding Cartan matrix.

Notice now that for $j \in \mathbb{Z}$ and $k=1,2, \ldots n$ the left-hand side of Eq. (43) has a fixed parity $\epsilon(k)(-1)^{j}$. Therefore, our $Y$ system decomposes into two independent systems, an even one and an odd one. Until this point, we have been considering merely the one of even type corresponding to our choice of $j+k \equiv 0 \bmod 2$; see Eq. (28) and (42). Now the question arises: What kind of patterns of entanglement are encoded into kinematic space by systems of the odd type?

Without the loss of generality, one may assume that [24]

$$
Y_{j+1, k}=Y_{j, k}^{-1}, \quad \text { whenever } \epsilon(k)=(-1)^{j},
$$

and as a result of this Eq. (43) can be rewritten as

$$
Y_{j+1, k}= \begin{cases}Y_{j, k} \prod_{l=1}^{n}\left(1+Y_{j, l}\right)^{b_{k l},}, & \text { if } \epsilon(k)=(-1)^{j+1}, \\ Y_{j, k}^{-1}, & \text { if } \epsilon(k)=(-1)^{j}\end{cases}
$$

Using the definitions (42) and (45), one can realize that formula (47) encapsulates information on the relationship between the entanglement measures of even and odd type in the form

$$
I_{j, k}-I_{j+1, k}=\frac{\mathfrak{c}}{3} \log t_{j, k} .
$$

This can be regarded as an $N>4$ generalization of Eq. (21) obtained for geodesic quadrangles. We also know that the entanglement measures $I_{j+1, k}$ should be related to area labels of some causal domain in $\mathbb{K}$.

Before clarifying the nature of these domains, let us illustrate the meaning of this even-odd duality for geodesic pentagons $(N=5, n=2)$. We use the recursion relation with the initial values

$$
\begin{aligned}
Y_{0,1} & =\frac{1}{u_{1}\left(1+u_{2}\right)}, \\
Y_{0,2} & =\frac{1+u_{1}+u_{1} u_{2}}{u_{2}}
\end{aligned}
$$

to obtain the set of labels featured in Fig. 10. One should start with the lower left part of the diagram having the green label of Eq. (49) and proceed in a counterclockwise fashion. In Fig. 10, the green labels are of even and the red ones are of odd type. By virtue of Eq. (45), the green labels then reproduce the $X_{j, k}$ variables of Eq. (29) of even type. [In arriving at this result, according to Eq. (44), $Y_{0,2}=Y_{5,1}$.] Notice also that using our recursion after completing a full counterclockwise circle in Fig. 10 the green and red labels are exchanged. This is in accord with Zamolodchikov periodicity, since the initial labels are

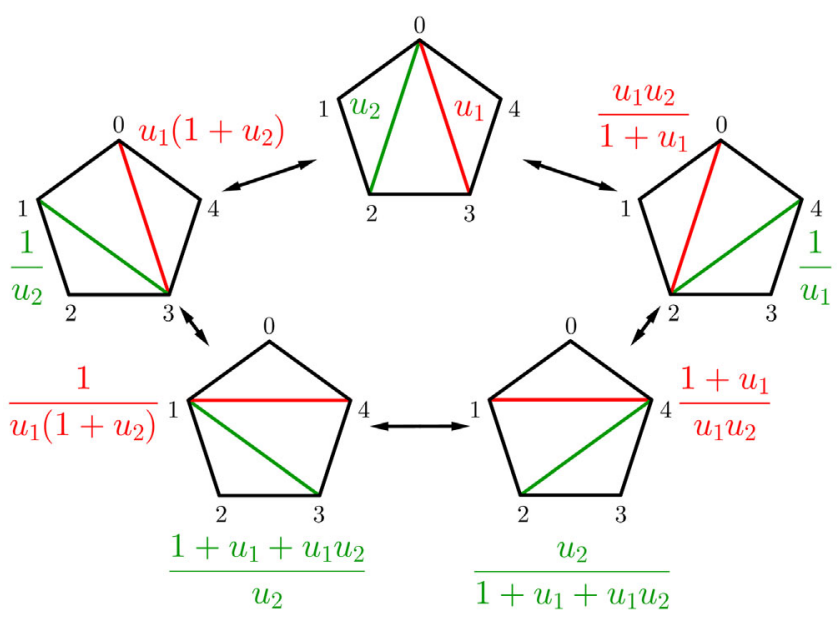

FIG. 10. Triangulations of a geodesic pentagon and the associahedron $\mathcal{K}^{2}$. Here the labels are shear coordinates $t_{j, k}$ related to the variables $X_{j, k}$ and $Y_{j, k}$ by Eq. (45). The green labels give rise to the explicit expressions of the even variables $X_{j, k}$ with $j+k \equiv 0 \bmod 2$ familiar from Eq. (29). The red ones give rise to the corresponding odd variables with $j+k \equiv 1 \bmod 2$. 
restored after two such cycles; i.e., one needs ten flips to get back. Recall also that this periodicity is just the discretized version of the periodicity of kinematic space in the $\theta$ coordinate.

In order to further clarify the meaning of the even-odd transition of Eq. (47), let us first record the obvious fact that it is related to flips of the corresponding quadrangles (see Fig. 10). Next, one should notice that for $\epsilon(k)=(-1)^{j}$ a transition of the type $Y \mapsto 1 / Y$ corresponds to $X \mapsto 1-X$. Now by plugging $j=n$ in Eq. (34) one obtains $X_{n+3, n}=1-X_{n+2, n}=\prod_{k=1}^{n} X_{k, k}$. Hence, the odd variable $X_{N, n}$ can be written as a product of even ones. However, one can even prove more. For example, for the $N=6$, $n=3$ case, apart from the corresponding relation $X_{6,3}=$ $X_{1,1} X_{2,2} X_{3,3}$ a set of relations of a more general kind hold. Indeed, one can check, for example, that

$$
X_{5,2}=1-X_{4,2}=X_{1,1} X_{2,2} X_{1,3} X_{6,2},
$$

and from Fig. 9 one discovers that the labels showing up in the product are the ones lying in the complement of the light cone of $X_{4,2}$. More precisely, since $X_{4,2}=X_{10,2}$ and the complements of the light cones are overlapping, if we avoid double counting, the labels are precisely the ones showing up in the complement of the light cone of $X_{4,2}$ in the central belt of $\mathbb{K}$. In fact, our example is just a special case of Proposition 4 in Ref. [27]:

$$
1-X_{j, k}=\prod_{\left(j^{\prime}, k^{\prime}\right) \in \overline{\mathcal{C}}_{j, k}} X_{j^{\prime}, k^{\prime}},
$$

where $1 \leq j \leq n+3,1 \leq k \leq n, j+k \equiv 0 \bmod 2$, and

$$
\overline{\mathcal{C}}_{j, k}=\left\{\left(j^{\prime}, k^{\prime}\right)|| k^{\prime}-k|<| j^{\prime}-j \mid\right\} .
$$

Since $1-X_{j, k}=X_{j+1, k}$, and the negatives of the logarithms of the $X$ 's are related to conditional mutual information, this is then in accord with the expectation that the $I_{j+1, k}$ 's are quantities related to a dual system of $X$ variables also satisfying the (33) recursion relations.

Using this result, Eqs. (22), (28), and (45), and the Brown-Henneaux relation, one obtains

$$
\frac{\ell_{\mathrm{AdS}}}{G_{\mathrm{N}}} \cdot \log \sinh \frac{\ell_{j, k}}{2}=I_{j, k}-\sum_{\left(j^{\prime}, k^{\prime}\right) \in \overline{\mathcal{C}}_{j, k}} I_{j^{\prime}, k^{\prime}} .
$$

Here $\ell_{j, k}$ is the distance in the bulk between the the geodesics anchored to the boundary regions $[(j-k) / 2$, $(j+k) / 2]$ and $[(j-k-2) / 2,(j+k+2) / 2]$. This equation shows how the difference between the conditional mutual information of even $\left(I_{j, k}\right)$ and odd types $\left(I_{j+1, k}\right)$ of the boundary are related to the corresponding geodesic lengths $\ell_{j, k}$ of the bulk or, alternatively, to the proper times $\Delta \tau_{j, k}$ elapsed between the two timelike separated vertices of the relevant causal diamonds in $\mathbb{K}$ (see the right-hand side of Fig. 4). Moreover, the sum in the right-hand side of Eq. (52) identifies $I_{j+1, k}$ of Eq. (48) as the sum of the areas of the causal diamonds lying in the complement of the light cone $\mathcal{C}_{j, k}$.

These considerations show that for large $n$ and in terms of $X$ variables the $\left(A_{n}, A_{1}\right)$-type Zamolodchikov systems of even and odd kind have an interesting entanglement interpretation in kinematic space. The $X_{j, k}$ of even systems are labeled by points $(j, k)$ in kinematic space and the odd ones with complements of light cones of such points. Correspondingly, there are two possible ways of scanning kinematic space by patterns of entanglement provided by causal domains associated to the $X_{j, k}$ 's. The two different ways are related by duality between points and the complements of their light cones. In the following section, we elaborate on this scanning process.

\section{SCANNING KINEMATIC SPACE}

We have seen that, by discretizing $\partial \mathbb{D}$ to $N$ regions and considering the corresponding gauge-invariant conditional mutual information of the CFT vacuum, from a seed pattern we can recursively generate area labels for a corresponding discretization of $\mathbb{K}$. The starting point of this generating process was a choice of a geodesic $\mathrm{N}$-gon taken together with a particular triangulation of the bulk $\mathbb{D}$. Now a natural question to be asked is: How this process of encoding entanglement patterns of the boundary into plaquette variables of $\mathbb{K}$ is depending on the choice of triangulations?

For a geodesic $N$-gon, we label the $N$ points on the boundary $\partial \mathbb{D}$ by the numbers $\{0,1, \ldots, N-1\}$. These points partition the boundary into $N$ regions $R_{0}, R_{1}, \ldots R_{N-1}$, where $R_{j}$ is a region between $j$ and $j+1$. Note that region $R_{N-1}$ between $N-1$ and $N \equiv 0 \bmod N$ is redundant, since it is fixed by the list $R_{0}, R_{1}, \ldots, R_{N-2}$. Given a geodesic $N$-gon, a diagonal is a geodesic between two nonadjacent boundary points. A partial triangulation is a collection of mutually noncrossing diagonals. A full triangulation or simply a triangulation is a partial triangulation with the maximal (i.e., $N-3$ ) number of diagonals. It is known [37] that for an $N \geq 3$-gon the number of (full) triangulations is given by the Catalan number $C_{N-2}=\frac{1}{N-1}\left(\begin{array}{c}2(N-2) \\ N-2\end{array}\right)$. Hence, for $N=3,4,5,6,7,8 \ldots$, we get the numbers $C_{N-2}=$ $1,2,5,14,42,132, \ldots$.

A list $\mathbf{R}_{0} \mathbf{R}_{1} \ldots \mathbf{R}_{N-1}$ will refer to the sequence of consecutive Ryu-Takayanagi geodesics of $\mathbb{D}$ anchored to the boundary regions $R_{0} R_{1} \ldots R_{N-1}$ forming a geodesic $N$ gon. In the following, we adopt the convention of referring to a diagonal by introducing a bracketing to this list. Hence, for $N=4$ the symbol $\left(\mathbf{R}_{0} \mathbf{R}_{1}\right) \mathbf{R}_{2} \mathbf{R}_{3}$ is a path of consecutive geodesic segments forming a quadrangle taken together with a diagonal connecting the boundary points 0 and 2. Notice that, since $\mathbf{R}_{\mathbf{3}}$ is redundant, by an abuse of notation one can omit the last entry and use the binary 
bracketing $\left(\left(\mathbf{R}_{0} \mathbf{R}_{1}\right) \mathbf{R}_{2}\right)$ instead. Indeed, the outer extra bracket can be regarded as a reference to connecting the boundary points 0 and 3, which is just $\mathbf{R}_{\mathbf{3}}$ elevated to the status of a "degenerate diagonal." The net result is that either of the symbols, the shorter $\left(\mathbf{R}_{0} \mathbf{R}_{1}\right) \mathbf{R}_{2}$ or the longer one $\left(\left(\mathbf{R}_{0} \mathbf{R}_{1}\right) \mathbf{R}_{2}\right)$, can be regarded as a mnemonic for a geodesic quadrangle taken together with a diagonal connecting the boundary points 0 and 2 .

Binary bracketings, like $\left(R_{0} R_{1}\right) R_{2}$ or $\left(\left(R_{0} R_{1}\right) R_{2}\right)$, have an obvious meaning related to the causal structure their corresponding boundary regions enjoy. Indeed, the regions showing up in any meaningful binary bracketing are either timelike or lightlike separated. We adopt the phrase that a set of regions contains compatible pairs if and only if no two of such pairs are spacelike separated. In our example, we have the regions $R_{0}, R_{1}$, and $R_{2}$ and the combined region $R_{0} R_{1}$; no two of them are spacelike separated. [An example for spacelike separated regions is provided by the pair $\left(R_{0} R_{1}, R_{1} R_{2}\right)$.] The alternative notation $\left(\left(R_{0} R_{1}\right) R_{2}\right)$ makes also reference to the extra region $R_{3}$. Of course, its inclusion into the list of regions is not destroying the compatibility structure.

To any meaningful binary bracketing of the boundary, one can associate a triangulation of the bulk. The corresponding binary bracketing of the list of consecutive geodesics $\mathbf{R}_{0} \mathbf{R}_{1} \ldots \mathbf{R}_{N-1}$ will be regarded as a representative of the particular triangulation in $\mathbb{D}$. Since geodesics are represented by points in kinematic space, a binary bracketing for the list of symbols $\mathcal{R}_{0} \mathcal{R}_{1} \ldots \mathcal{R}_{N-1}$ gives rise to a collection of points in $\mathbb{K}$ forming a causal pattern. For example, it is easy to check that the bracketing $\left(\mathcal{R}_{0}\left(\mathcal{R}_{1}\left(\ldots \mathcal{R}_{N-1}\right)\right)\right)$ gives rise to two triangular shapes contained in the upper and lower light cones of the point $N-1$. The points $\mathcal{R}_{0} \mathcal{R}_{1} \ldots \mathcal{R}_{N-1}$ are lightlike separated, and they lie on the right-hand side of the corresponding light cones. An illustration of this for $N=6$ can be seen in the top pattern in Fig. 13. Further examples for causal patterns can be seen in Figs. 6 and 8. In the first case, the causal pattern is the one answering the boundary binary bracketing of regions of the form $\left(\left(\left(R_{0} R_{1}\right) R_{2}\right) R_{3}\right)$ and in the second case of the form $\left(\left(\left(R_{0} R_{1}\right)\left(R_{2} R_{3}\right)\right) R_{4}\right)$.

For $N=4$, the two possible bulk triangulations are shown in Fig. 5. In the boundary using the binary bracketing, one can refer to them as $\left(\left(R_{0} R_{1}\right) R_{2}\right)$ and $\left(R_{0}\left(R_{1} R_{2}\right)\right)$. We also know that to the two possible diagonals one can associate shear coordinates $u=t_{12,30}$ and $1 / u$, which, in turn, encode the conditional mutual information of Eqs. (26a) and (26b). A change from one of the diagonals of a particular geodesic quadrangle to the other one is a flip. Since diagonals are taken together with shear labels, we see that for the $N=4$ case a flip is also associated with the change $u \mapsto 1 / u$. In terms of the conditional mutual information related by flips, the shear is expressed by Eq. (21). In the following, we will call the flips of causal patterns answering the flip in the corresponding triangulation a mutation of a causal pattern. An example for a mutation of a causal pattern can be seen in the lower part in Fig. 5.

Now for $N \geq 3$ one can define a convex polytope of dimension $N-3$ called the associahedron $\mathcal{K}^{N-3}$, such that for every $d=0,1, \ldots N-3$ there is a one-to-one correspondence between its $d$-dimensional boundaries and the $d$-diagonal partial triangulations of the geodesic $N$-gon $[32,38]$. Here by boundary we mean a boundary of the polytope of any codimension. Moreover, a codimension $d$ boundary $B_{1}$ and a codimension $d+l$ boundary $B_{2}$ are adjacent if and only if the partial triangulation of $B_{2}$ can be obtained by the addition of $l$ diagonals to the partial triangulation of $B_{1}$. The vertices of the associahedron correspond to the (full) triangulations. The labeling of the vertices of $\mathcal{K}^{2}$ and $\mathcal{K}^{3}$ by such triangulations can be seen in Figs. 10 and 11. Notice that adjacent triangulations are related by a flip. The associahedron boundaries related to partial triangulations are illustrated for $\mathcal{K}^{3}$ in Fig. 12.

For $N=5$, the list of all possible binary bracketings of boundary regions is as follows:

$$
\begin{array}{lll}
\left(\left(R_{0}\left(R_{1} R_{2}\right)\right) R_{3}\right), & \left.\left(\left(R_{0} R_{1}\right) R_{2}\right) R_{3}\right), & \left(\left(R_{0} R_{1}\right)\left(R_{2} R_{3}\right)\right), \\
\left(R_{0}\left(\left(R_{1} R_{2}\right) R_{3}\right)\right), & \left(R_{0}\left(R_{1}\left(R_{2} R_{3}\right)\right)\right) . &
\end{array}
$$

The reader can check that the pentagonal-like arrangement of this list of binary bracketings encoding compatible boundary regions corresponds to the pentagonal arrangement of bulk triangulations in Fig. 10. Moreover, in kinematic space these triangulations give rise to five causal patterns. These patterns arise from the middle top triangulation in Fig. 10 after representing it in $\mathbb{K}$ and then proceeding in a clockwise fashion. Explicitly, it is easy to show that these patterns arise from the one in Fig. 6 by a

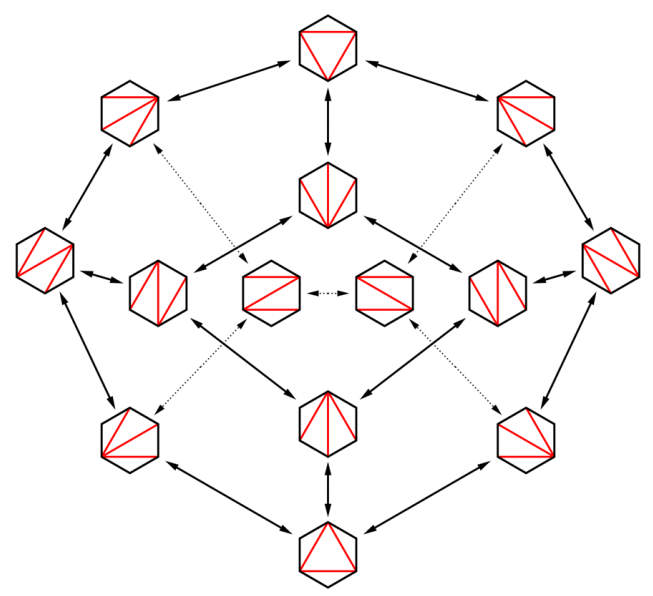

FIG. 11. Triangulations of a geodesic hexagon and the associahedron $\mathcal{K}^{3}$. The vertices of the associahedron are labeled by triangulations of $\mathbb{D}$. Adjacent triangulations are related by a flip of one of the diagonals belonging to a geodesic quadrangle. 


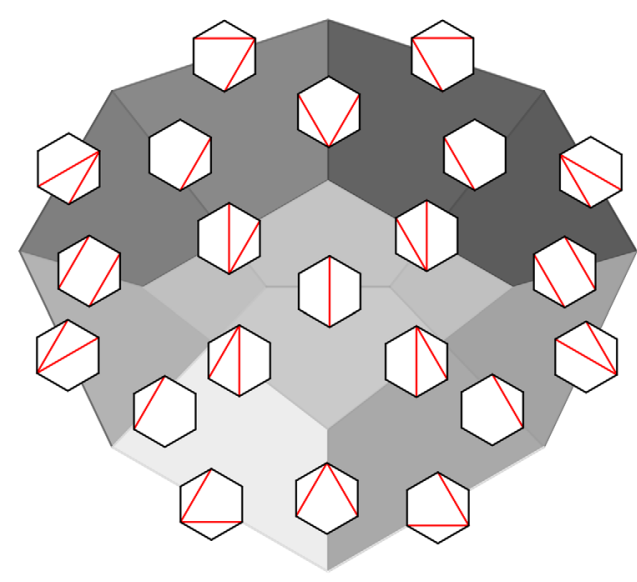

FIG. 12. The associahedron $\mathcal{K}^{3}$ with its edges and faces labeled by partial triangulations of the geodesic hexagon.

cyclic shift to the left. Then this sequence of mutations of causal patterns scans the central belt of kinematic space.

Let us now observe that the distribution of closing brackets uniquely determines the binary bracketing [39-41] and, hence, the causal pattern in $\mathbb{K}$. Now if in the corresponding opening brackets we choose a constant distribution just one before each symbol $R_{j}, j=0,1, \ldots N-2$, what we get is a right bracketing. The right bracketings in the $N=5$ case are as follows:

$$
\begin{array}{lll}
R_{0}\left(R_{1}\left(R_{2}\right)\right)\left(R_{3}\right), & R_{0}\left(R_{1}\right)\left(R_{2}\right)\left(R_{3}\right), & R_{0}\left(R_{1}\right)\left(R_{2}\left(R_{3}\right)\right), \\
R_{0}\left(R_{1}\left(R_{2}\right)\left(R_{3}\right)\right), & R_{0}\left(R_{1}\left(R_{2}\left(R_{3}\right)\right)\right) .
\end{array}
$$

Each right bracketing can be encoded into an $(N-2)$-vector $v \equiv\left(v_{1}, v_{2}, \ldots, v_{N-2}\right)$ as follows $[40,41]$. For $\mu=1,2, \ldots N-2$, we have $v_{\mu}=\nu$ if and only if the opening bracket before $R_{\mu}$ closes after $R_{\nu}$. For example, for the $N=5$ case, we get the following set of 3-vectors:

$$
(2,2,3), \quad(1,2,3), \quad(1,3,3), \quad(3,2,3), \quad(3,3,3) .
$$

Now one can prove $[39,40]$ that the components of our $(N-2)$-vectors, comprising a space $\mathcal{V}_{N-2}$, are positive integers $\leq N-2$ and they satisfy the following two constraints: $v_{\mu} \geq \mu$ and $v_{\mu} \geq \nu>\mu$ implies $v_{\mu} \geq v_{\nu}$ for $\mu=$ $1,2, \ldots N-2$. The latter condition captures the fact that two different pairs of brackets do not overlap; in other words, no spacelike separation of boundary regions is allowed. One can then prove that the number of such $(N-2)$-vectors is $C_{N-2}$; i.e., it equals the number of (full) triangulations of our geodesic $N$-gon. In this way, we managed to characterize the space of causal patterns by a set of $(N-2)$-vectors of cardinality $C_{N-2}$.

Now the important point we would like to emphasize is that the space of causal patterns $T_{N-2}$ in $\mathbb{K}$ is also equipped with a partial order. Moreover, the corresponding partially ordered set $\left(T_{N-2}, \leq\right)$ of causal patterns is isomorphic to a lattice: the Tamari lattice $[33,39]$. Indeed, let us identify the space of all binary bracketings with $T_{N-2}$. Then our $N-1$ boundary regions $R_{j}$ (recall that we have not included into the list the region $R_{N-1}$ ) can be ordered according to the semiassociativity rule [33,39,41]: $(A B) C \mapsto A(B C)$. Notice that as far as entanglement is concerned a particular bracketing of $N$ boundary regions can be interpreted as a choice of context in which our boundary subdivision should be considered. In this picture, applying the semiassociative rule in $\partial \mathbb{D}$ is just the elementary change in the space of contexts. Notice that there is an ambiguity here in whether we choose the left or right ordering of brackets in the semiassociative rule. Our choice is the one compatible with the rule represented in $\mathbb{D}$ by the usual flip in the corresponding geodesic quadrangle. Notice also that our flip, in turn, is represented by a causal flip, i.e., a mutation of a starting pattern to another one in $\mathbb{K}$. Now for two causal patterns $\mathcal{T}_{1}, \mathcal{T}_{2} \in T_{N-2}$ we define $\mathcal{T}_{1} \leq \mathcal{T}_{2}$ if and only if $\mathcal{T}_{1}$ can be transformed into $\mathcal{T}_{2}$ by the repeated application of our semiassociativity rule. One can then identify $T_{N-2}$ with the set of $(N-2)$-vectors satisfying our aforementioned two constraints [40], and the partial order in this set of vectors is just componentwise comparison. Hence, for example, for the $N=5$ case, one has $(3,3,3) \geq(3,2,3) \geq$ $(2,2,3) \geq(1,2,3)$ and $(3,3,3) \geq(1,3,3) \geq(1,2,3)$; however, the vectors within the pairs $((1,3,3),(3,2,3))$ and $((1,3,3),(2,2,3))$ are not related. These considerations establish the Tamari lattice structure for $T_{3}$, i.e., the space of causal patterns for $N=5$.

The Tamari lattice $T_{4}$ of causal patterns in $\mathbb{K}$ for $N=6$ can be seen in Fig. 13. Generally, there is a maximal and minimal element of the lattice $T_{N-2}$. In the $(N-2)$-vector notation, they correspond to the vectors $(N-2, N-2, \ldots$, $N-2, N-2)$ and $(1,2, \ldots, N-1, N-2)$. In terms of RyuTakayanagi geodesics, the corresponding diagonals give rise to "fans" emanating from the points $N-1$ and 0 , respectively. Clearly, the corresponding causal patterns are just pairs of past (future) light cones emanating from the points $\mathcal{R}_{N-1} \in \mathbb{K}$ or $\mathcal{R}_{0} \in \mathbb{K}$ lying inside the past (future) light cones of the points (degenerate geodesics) $N-1$ and 0 lying in past (future) infinity of $\mathrm{dS}_{2}$. For an illustration of this for $N=6$, see Fig. 13. Notice that each of the causal patterns is uniquely determined by $N-3$ points in $\mathbb{K}$ corresponding to the diagonals in $\mathbb{D}$. The reader can verify this statement by looking at the causal patterns and identifying their corresponding points in Figs. 6 and 8 .

In the following, we adopt the convention of picturing these points inside the past light cone of the point $N-1$. More precisely, what we need is a part of this past light cone which forms an isosceles right angular triangular region constructed as follows. Define the central belt of the grid, depicted for $N=5,6$ in Figs. 7 and 9, as the region obtained by removing the plaquettes labeled by the variables $X_{j, 0}$ and $X_{j, n+1}$ from the lattice. Then the triangular region is that part of the lattice with points lying 


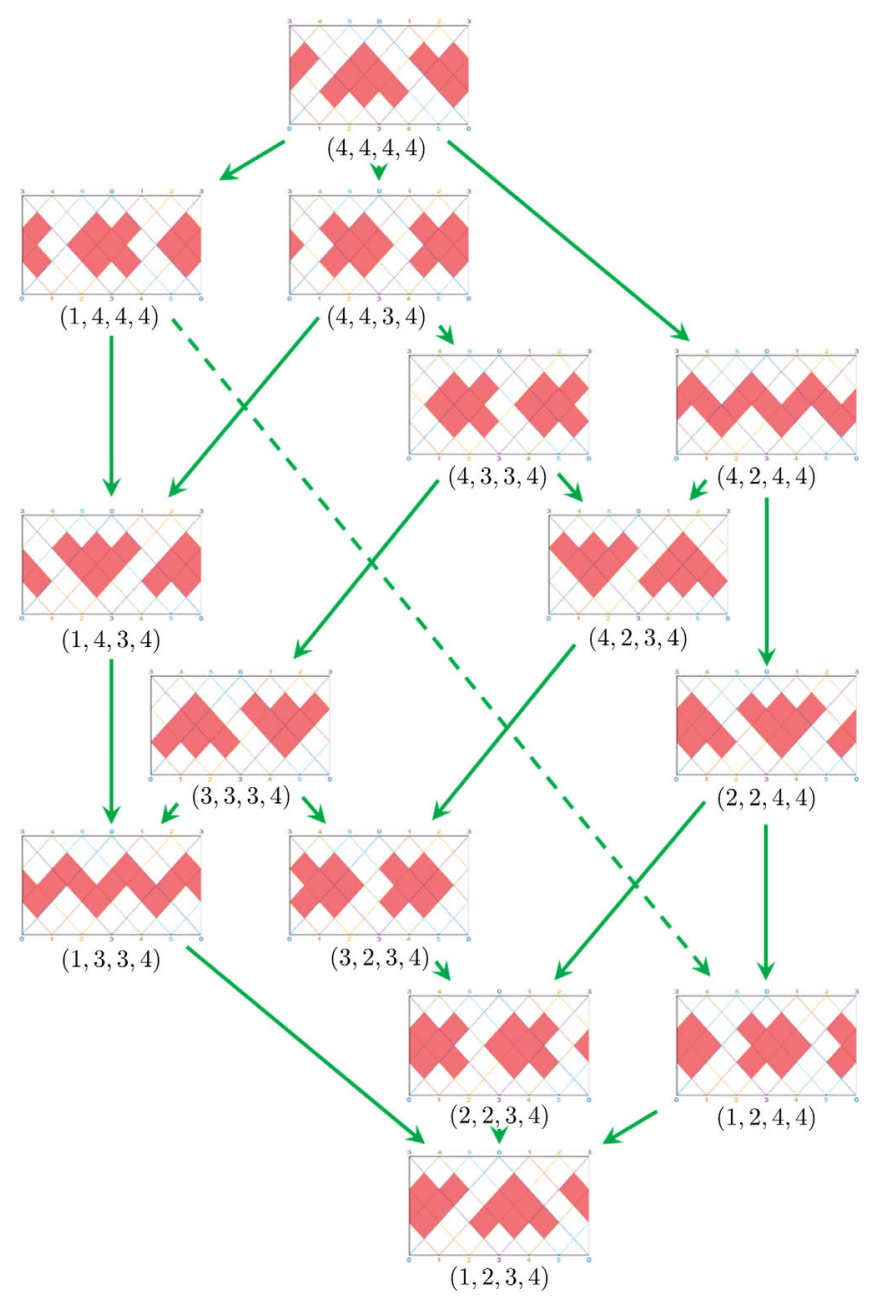

FIG. 13. The Tamari lattice of causal patterns $\left(T_{4}, \leq\right)$ in kinematic space. Each causal pattern is labeled by a vector as an element of $\mathcal{V}_{4}$ defined in the text. In terms of these vectors, the partial order on $T_{4}$ is represented by componentwise comparison. Notice that the causal patterns show up in dual pairs, their orientation reflecting the twisted boundary condition of Eq. (36) giving rise to Zamolodchikov periodicity.

inside and on the past light cone of $\mathcal{R}_{N-1} \in \mathbb{K}$, which has its base formed by the boundary of the central belt.

The evolution of causal patterns can be characterized as a walk of $N-3$ distinguishable particles subject to a set of rules (to be described below) in a triangular region of the past light cone of the point $\mathcal{R}_{N-1}$. (The reader can visualize the following considerations by looking at the triangular region cut out of the light cone of the point $\mathcal{R}_{5}$ for the $N=6$ case in Fig. 14.) The evolution process starts by putting all of the $N-3$ particles on the right-hand side of our triangular region. This means that all of the particles are lined up on the point curve of the point $N-1$. We identify the particle labels $1,2, \ldots N-3$ with the label of the other point curve the particle is lying on.

Now we define a coordinate grid in our triangular region with left- (right-) moving light cone coordinate lines by

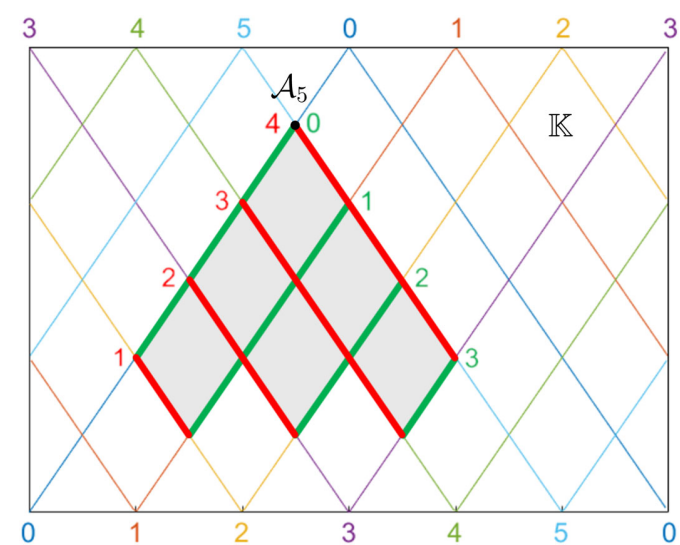

FIG. 14. The triangular region of kinematic space where the $N=6$ random walk takes place.

representing them by red (green) segments of the relevant point curves. The right-moving segments are on the point curves with labels $0,1, \ldots N-3$, and the left-moving ones are on the ones with labels $2,3, \ldots, N-1$. Let us now relabel the left moving grid lines as $1,2, \ldots N-2$. Let us now focus on the red and green segments with identical labels: $1,2, \ldots, N-3$. The green and red parts of these curves are exchanged after reflecting from the boundary of the triangular region. We call these $N-3$ curves the "reflected point curves." Moving one unit down along the green part will be called a green move. Similarly, moving one unit $u p$ along a red part will be called a red move. Notice that, since such green and red lines are labeled from $1,2, \ldots N-3$, they are naturally ordered. Then particles executing moves are prioritized according to the magnitude of the curve on which they are located. For a particle executing red moves of a certain (red) priority, an intersecting a green curve will be called occupied if it already features another particle with a larger (red) priority.

Let us call the initial configuration the one when the $N-3$ particles are lined up on the right-hand side of our triangular region and the final one when all of them are lined up on the left-hand side of the region. (See the top and bottom configurations of the $N=5$ case in Fig. 15.) These configurations correspond to the maximal and minimal elements of the Tamari lattice $T_{N-2}$. Now the evolution of the initial configuration starts with a random choice of one from the $N-3$ particles. The next step involves applying to this particle a sequence of green moves followed by a sequence of red ones with the particular sequences determined by the following set of rules. (i) If the point curve of the particle is not blocked by any other one in the green direction, then the particle walks, employing green moves to reach the boundary, and then switches there to execute red moves. (ii) Any walk of a particle executing red moves terminates at the crossing point with an occupied green curve. In the absence of any obstruction for red moves, the particle will reach the final (leftmost) position. (iii) A particle executing green moves blocked by another particle 


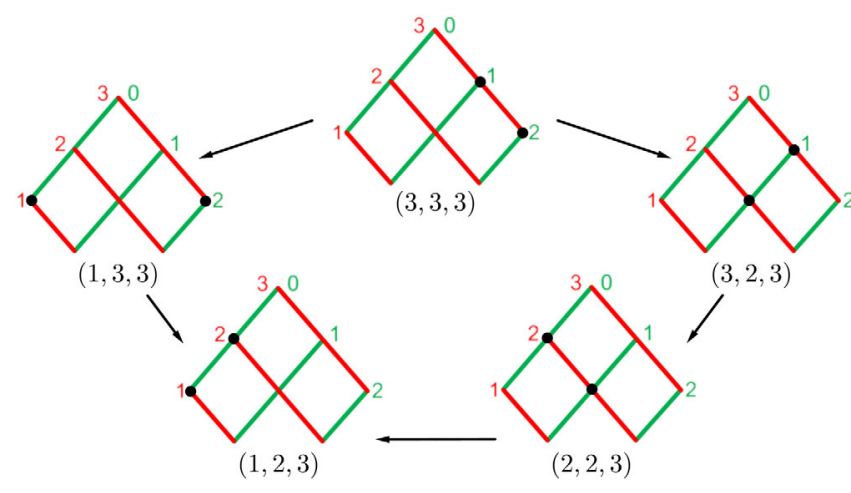

FIG. 15. The walk for $N=5$ in kinematic space. Note that in this walk two particles are moving, represented by the two black bullets showing up in these diagrams. In fact, there should be a third bullet at the tip of the light cone, which corresponds to a particle never changing its position. However, we omitted this particle from our diagrams. Since the position of this particle, i.e., the position of $\mathcal{R}_{4}$, is fixed, it produces at each step of the walk the same number (namely, 3$)$ for the $(3 \equiv 0 \bmod 3)$ third component of the vector in $\mathcal{V}_{3}$.

switches to executing red moves from the meeting point. These rules will then result in a new configuration. Now apart from the particle having just finished its walk, we have $N-4$ new ones to choose from for the continuation of the evolution. After choosing randomly at each step, the process terminates at the final configuration. The simplest case of the random walk associated with $T_{3}$ is illustrated in Fig. 15.

It is easy to check that this collection of rules to be applied in $\mathbb{K}$ corresponds to the usual flip operation of diagonals of geodesic quadrangles in $\mathbb{D}$. However, since the location of the $N-3$ particles also encodes the corresponding causal pattern taken together with their area (conditional mutual information) labels, the new representation also encapsulates a visualization of patterns of entanglement of the CFT vacuum in two-dimensional de Sitter spacetime. Another virtue of this geometric approach is that we can immediately read off the components of the $(N-2)$-vectors as some sort of coordinates identifying a particular causal pattern.

In order to show that the $(N-2)$-component vectors are indeed nicely displayed in our triangular regions of $\mathbb{K}$ where the random walk enfolds, first observe that the $(N-2)$ th component always equals $N-2$. This corresponds to the omnipresent outer bracket in the binary bracketing or, alternatively, to our regarding the geodesic $\mathbf{R}_{N-1}$ as a degenerate diagonal. In $\mathbb{K}$, this reflects the fact that the point $\mathcal{R}_{N-1}$ being the tip of the light cone plays a special role in our considerations. Hence, one has to focus only on the first $N-3$ components of our vector.

The next step is to notice that the reflected point curves for the corresponding $N-3$ particles are showing up in a dual role. Indeed, the green label of these curves can be used to identify the label of the component, and the red label can be used to identify the value in question of that component. For example, for the topmost pattern in Fig. 15, all of the particles having a red label equal 3, and they are on the reflected point curves: number 1 and 2. As another example, one can consider the leftmost pattern. Here the red labels give 1 and 3 , and the first one can be associated with point curve number 1 and the other with number 2 .

One can obtain similar arrangements with three particles for the $N=6$ case. They determine the causal patterns in Fig. 13. For this case, the whole collection of 4-vector labels for $T_{4}$ can be seen in Fig. 13. Using these labels, the reader can check that the partial order structure of the causal patterns holds. These labels also fix the positions of the three distinguishable particles in the region in Fig. 14 (not shown in Fig. 13). This produces a figure for $N=6$ similar to the detailed one in Fig. 15 of $N=5$.

Finally, we note that, thanks to the explicit relationship found here between causal patterns and conditional mutual information of boundary regions, the Tamari order $\left(T_{N-2}, \leq\right)$ also introduces a partial order between patterns of entanglement. The representation of collections of boundary regions as some sort of "space of contexts" equipped with binary bracketings is connected to the original setup where the Tamari order has shown up. Now it is also known that boundary regions enjoy a causal order based on the containment relation. A new twist to this idea is the finding that the Tamari ordering is yet another type of ordering present in the boundary. It is a partial order which for a fixed $N$ relates different contexts of $N$-fold partitions. This context space equipped with this partial order is also associated with a set of gauge-invariant entanglement measures $I_{j, k}, j=1,2, \ldots N$, $k=1,2, \ldots N-3$. These structures working in concert then introduce a new type of holographic entanglement hierarchy which is connected to a corresponding evolution governed by the $A_{N-3}$ cluster dynamics. The exploration of the physical implications of these structures within a holographic context deserves further attention.

\section{CONCLUSIONS}

\section{A. Summary}

Using the principle of holography and following the ideas introduced by one of us in a previous paper [17], in this work we elaborated on the issue of how patterns of entanglement of the boundary are encoded into the classical geometry of the bulk. In this paper, we have chosen the simplest setup, namely, the $\mathrm{AdS}_{3} / \mathrm{CFT}_{2}$ correspondence, by restricting our attention to the static slice and on patterns of entanglement of the CFT vacuum. Apart from the basic spaces featuring any holographic consideration, namely, the bulk and its boundary, we have also made use of kinematic space, which is usually regarded as an intermediary between the bulk and boundary [7]. However, apart from translating between the language of quantum information of the boundary $\partial \mathbb{D}$ to the language of geometry of 
the bulk $\mathbb{D}$, the kinematic space $\mathbb{K}$ is also an interesting space in its own right. Namely, in the static case, $\mathbb{K}$ is just the two-dimensional de Sitter space $\mathrm{dS}_{2}$, which provides a very simple example of an emergent spacetime structure to be understood in quantum entanglement terms. In fact, it is a vacuum solution of Einstein's equations; hence, its connection to the CFT vacuum seems to be of basic importance.

We have shown that one can understand $\mathrm{dS}_{2}$ as a space of causal patterns encoding entanglement information of the CFT vacuum. The entanglement information is provided by a set of cutoff-independent conditional mutual information associated with binary bracketings of partitions of the boundary into $N$ subregions. The set of all such bracketings defines the space of contexts of $N$ subregions, with cardinality $C_{N-2}$. The causal patterns are also defined by $C_{N-2}$ configurations of $N-3$ pairwise compatible points in $\mathrm{dS}_{2}$. Such causal patterns consist of $(N-2)(N-3) / 2$ causal diamonds with their area labels related to conditional mutual information. These labels are also connected to cluster variables. Mutations of causal patterns then turn out to be related to the coefficient dynamics $[23,24]$ of a cluster algebra of type $A_{N-3}$.

Mutations consist of elementary flips. At the boundary level, they are represented by applying the semiassociative law of right or left shifts of binary bracketings. This corresponds to a flip in the space of contexts. At the bulk level, they are represented by flips between the two possible diagonals of a geodesic quadrangle. This corresponds to a flip in the space of triangulations of geodesic $N$-gons. At the kinematic space level, a flip is represented by an elementary step taken by one of $N-3$ particles executing a random walk on the lattice inside a triangular region provided by the past light cone of a distinguished point.

As is well known in the mathematics literature, the coefficient dynamics of an $A_{n}$ cluster algebra is the dynamics of cross ratios. Cross ratios are related to shear coordinates $t_{j, k}$, which define coordinates for Teichmüller space of $\mathbb{D}$ with $N=n+3$ marked points. Then after a flip in $\mathbb{D}$ these coordinates are transformed according to the coefficient dynamics of the $A_{n}$ cluster algebra. This dynamics is related to a Zamolodchikov $Y$ system of type $\left(A_{n}, A_{1}\right)$. In Eq. (33), we have rewritten this dynamics in terms of the $X_{j, k}$ plaquette variables $(j+k \equiv 0 \bmod 2)$, which are defined in Eq. (28) and related to the usual $Y_{j, k}$ variables and shears via Eq. (45).

We have shown that, apart from $X_{j, k}$ with $j+k \equiv 0 \bmod$ 2 , there are also dual variables with $j+k \equiv 1 \bmod 2$. It turned out that the former set of variables is associated with points $(P)$ and the latter with complements of the light cones of such $P$ 's $(\bar{C})$ in $\mathrm{dS}_{2}$. Moreover, we demonstrated that between the $P$ and $\bar{C}$ descriptions the interesting (52) relation holds. Indeed, the difference between the corresponding mutual information is related to the geodesic length (proper time) between the timelike separated points in $\mathrm{dS}_{2}$ defining the relevant causal diamond labeled by $P$. This result was based on the fact that the nodes of the $A_{n}$ Dynkin diagram are of two types: even or odd. Note that since any Dynkin diagram is a tree [24]; this result generalizes for other type of cluster algebras and cluster dynamics, possibly associated with other boundary quantum states different from the vacuum [17].

For fixed $N$, the space of causal patterns $T_{N-2}$ provides a set of regions covering $\mathrm{dS}_{2}$. The collection of such patterns of cardinality $C_{N-2}$ can be identified with the vertices of the associahedron $\mathcal{K}^{N-3}$. The boundaries of the associahedron of different dimensions correspond to different partial triangulations. In particular, the collection of facets of cardinality $N(N-3) / 2$ can be identified with the collection of Ryu-Takayanagi geodesics corresponding to diagonals. We have also established on the space of causal patterns $T_{N-2}$ a partial order, rendering this space a lattice $\left(T_{N-2}, \leq\right)$ isomorphic to the Tamari lattice. This space is also isomorphic to the set $\mathcal{V}_{N-2}$ of $(N-2)$-vectors with their components taken from $\mathbb{N}$ satisfying the constraints: For $\mu=1,2, \ldots, N-2$, we have $\mu \leq v_{\mu} \leq N-2$, and the one that $\mu \leq \nu \leq v_{\mu}$ implies $v_{\nu} \leq v_{\mu}$. The partial order in $T_{N-2}$ is then rephrased in $\mathcal{V}_{N-2}$ as a componentwise comparison $[39,40]$. We demonstrated that the elements of $\mathcal{V}_{N-2}$ used as coordinates for the elements of $T_{N-2}$ are nicely visualized in the triangular region inside the past light cone of the point $\mathcal{R}_{N-1}$. The first $N-3$ components identify $N-3$ points as the locations of the particles subject to the random walk explained in the previous paragraph.

We observed that the Tamari order defines a natural partial ordering on the space of contexts of the boundary. It is well known that boundary regions enjoy a causal order based on the containment relation [7]. Now we have revealed that there is yet another type of ordering present in the boundary. It is a partial order which for a fixed $N$ relates different contexts (represented by different binary bracketings) of $N$-fold partitions of the boundary. The space of contexts with this causal ordering is also characterized by the set of mutual conditional information $I_{j, k}$, $j=1,2, \ldots N, k=1,2, \ldots N-3$. This partial order introduces a new type of holographic entanglement hierarchy which is to be taken together with a corresponding evolution governed by the $A_{N-3}$ cluster dynamics. The physical meaning of these notions in a holographic context is yet to be explored.

\section{B. Outlook}

Let us finally comment on possible ramifications of our work connecting it to currently explored research topics. First of all, notice that, as $N$ goes to infinity, we are getting finer grids on $\mathbb{K}$; hence, the recursion of Eq. (33) should, in the continuum limit, boil down to some sort of field equation, with the $X_{j, k}$ variables giving rise to fields $X(\theta, \alpha)$. Because of the (28) relation, this approach will 
produce an entanglement field $I(\theta, \alpha)$ for the conditional mutual information. What is this field equation?

It is well known that the recursion valid for $Y$ systems can be regarded as the discretization of Liouville's equation $[42,43]$. So, following the ideas in these papers, one is expecting that the relevant field equation is just the Liouville equation. However, there are statements [44] in the literature that, though it is easy to see that a formal discretization of the Liouville equation indeed yields an $Y$ system identical to the one of our Eq. (43), it is not a $Y$ system satisfying Zamolodchikov periodicity. Since in our setup Zamolodchikov periodicity is inherently connected to periodicity of kinematic space, in order to answer this question some adaptation of the ideas of these papers should be desirable. The equation in question should be a wave equation in $\mathrm{dS}_{2}$ with some source term related to $I(\alpha, \theta)$. However, in order to properly implement Zamolodchikov periodicity, one also has to take into account the continuum version of Eq. (36), i.e., a twisted boundary condition.

Notice that our problem is related to the observation $[9,16]$ that the one region entanglement entropy is a Liouville field. Then one can conjecture that the entanglement dynamics of the $\mathrm{CFT}_{2}$ can be described by some sort of $(1+1)$-dimensional gravity. Though pure Einstein gravity is trivial in $1+1$ dimensions, however, JackiwTeitelboim (JT) gravity $[45,46]$ is not. This idea has already been followed in the literature $[15,47]$ with the conclusion that the dynamics in $\mathbb{K}$ is indeed gravitational and is described by the JT theory. Now one of the equations in JT theory is just the Liouville equation for $S$ of the form [15]

$$
\partial_{u} \partial_{v}\left(\frac{12}{\mathfrak{c}} S\right)=\frac{2}{\delta^{2}} e^{-(12 / \mathfrak{c}) S},
$$

where $\delta$ is an UV cutoff and $u, v$ is related to $\alpha, \theta$ by Eq. (7). Hence, a naive guess for the corresponding equation to be satisfied by $I(\theta, \alpha)$ would be a cutoff-independent analog of this equation with $S(\theta, \alpha)$ replaced by $I(\theta, \alpha)$. Moreover, one should ensure also that the twisted boundary condition $I(\theta+\pi, \pi-\alpha)=I(\theta, \alpha)$ holds.

There is yet another very interesting connection of our work with the recent research on the scattering amplitudes concerning the biadjoint $\phi^{3}$ theory [32]. According to this theory, the tree-level scattering amplitudes are given by the geometry of the associahedron in a space also called by the authors kinematic space. Moreover, in this seemingly different context, quite naturally, for the tree-level calculations a cluster algebra of type $A_{N-3}$ shows up. In this approach, kinematic space is the space of linearly independent Mandelstam invariants $s_{i j}=2 p_{i} \cdot p_{j}$, where $i, j=$ $1,2, \ldots N$ and the $p_{i}$ are massless momenta. Since the $s_{i j}$ are not independent, it turns out that the dimension of kinematic space is just the same as the number of facets of the associahedron $\mathcal{K}^{N-3}$, which is $N(N-3) / 2$, the same as the number of Ryu-Takayanagi geodesics corresponding to diagonals in the triangulations of our bulk. Another basis of the same cardinality can be used for the Mandelstam invariants by the planar variables (propagators) $\chi_{i, j}$. Identifying the momenta as $N$ marked points on $\partial \mathbb{D}$, one realizes that, just like the entanglement entropies of boundary regions anchored to the diagonals that are related to the (regularized) geodesic lengths, such planar variables are related to the lengths along the diagonals between $i$ and $j$. Moreover, from our previous paper [17], it is known that for the geodesics comprising the sides of the geodesic $\mathrm{N}$-gon by a suitable choice of regulators the entanglement entropies can be scaled away. This can be done thanks to a gauge degree of freedom encoded into the geometry of the space of horocycles and the fact that the associated lambda lengths can be related to the entanglement entropy [17]. This corresponds to the fact that the planar variables $\chi_{i, i+1}$ and $\chi_{1, n}$ are vanishing; i.e., they have length zero.

Now recall Eq. (45) of Ref. [17]. According to this equation, the regularized entanglement entropies of boundary regions are related to the lambda lengths of the corresponding geodesics. According to the previous paragraph, lambda lengths for diagonal boundary regions between nonadjacent $i$ and $j$ can be related to the planar variables $\chi_{i, j}$. However, for regularized entanglement entropies, lambda lengths can be negative. Now if we recall the positivity condition Eq. (3.4) of Ref. [32], one realizes that this condition can be interpreted as a constraint of restricting our attention to the physical branch of regularized entropies that are non-negative. Then one can entertain an analogy between $\chi_{i j}$ and the entanglement entropies belonging to the physical branch. In order to match the dimension of $\mathcal{K}^{N-3}$ and the dimension of the physically relevant subspace of kinematic space, the authors of Ref. [32] invoke a positivity constraint of their Eq. (3.6). Our observation in this respect is that if the planar variables are interpreted as objects analogous to entanglement entropies, then this positivity constraint is just our strong subadditivity relation of Eq. (13), which is now satisfied automatically. In this picture, the quantities $c_{i j}$ of Ref. [32] are analogous to the conditional mutual information, the main actors of our paper. Note that strong subadditivity is a highly nontrivial constraint in entanglement theory having a somewhat hard to swallow operational meaning [20] related to entanglement monogamy. However, its geometric meaning is quite transparent in $\mathbb{K}$. In Ref. [17], we managed to relate boundary entanglement to scattering data in an elementary way; now by working out the fine details of the analogy just revealed suggests that the analogy between scattering amplitudes and entanglement is probably much deeper.

In this paper, we have merely considered the static slice of $\mathrm{AdS}_{3}$. How can we generalize our considerations for a more general setup? Notice in this respect that the basis of our considerations was the link between the conditional mutual 
information associated with the boundary and geodesic quadrangles of a static slice of the bulk coming from putting four distinguished points on the boundary. Clearly, when one considers four points of more general locations, what one gets is a tetrahedron. Its six edges are geodesics. Now it is known that there are ideal tetrahedra [48] with vertices $a, b, c$, and $d$ labeled by real coordinates with its volume depending on the cross ratio in the following manner:

$$
\operatorname{Vol}(a b c d)=L\left(\frac{(a-c)(b-d)}{(b-c)(a-d)}\right)-\frac{\pi^{2}}{6}
$$

where $L(x)$ is the Rogers dilogarithm [choosing a sheet in which $|\operatorname{Vol}(a b c d)|$ has minimal value]. One can then speculate to relate conditional mutual information of boundary regions to such volumes. Moreover, in this case, instead of using triangulations of $\mathbb{D}$, one has to use triangulations of $\mathrm{AdS}_{3}$ by tetrahedra. Then following Refs. [48,49] the analogs of our $X$ and $Y$ variables should be associated to tetrahedra. Moreover, Zamolodchikov's $Y$ systems of type $\left(A_{N-3}, A_{1}\right)$ should show up naturally in this treatment [48]. However, since in this more general case kinematic space consists of two copies of $\mathrm{dS}_{2}$ spaces [8,10], at first sight it is not obvious how to generalize our findings.

Finally, what about other states of the CFT, different from the vacuum? As far as this question is concerned, we remind the reader that the original context of mathematics where cluster algebras made their debut was the Teichmüller theory of marked Riemann surfaces $[18,19,23,24]$. Such surfaces can be uniformized by factorizing $\mathbb{D}$ by Fuchsian groups $\Gamma$ giving rise to tilings of $\mathbb{D}$ by fundamental domains with their sides identified by certain group elements. This is the setting where cluster algebras associated with Dynkin diagrams, and also others than our one of type $A_{N-3}$, appear. Now $\mathbb{D}$ can be embedded into $\mathrm{AdS}_{3}$ and the action of $\Gamma$ can be extended [50,51] to $\mathrm{AdS}_{3}$. This construction has the well-known physical interpretation of obtaining multiboundary wormhole solutions [51,52] of extremal and nonextremal type generalizing the Banados-Teitelboim-Zanelli black hole [53]. Now, the holographic dual of such multiboundary wormhole solutions produces an interesting class of CFT states amenable for future study. Such states apparently have entanglement patterns worth studying in kinematic space $[14,22]$, in a spirit similar to the one of this paper. One can then expect that cluster algebras associated with the classical Dynkin diagrams (quivers) appear naturally in this context. Moreover, since such a type of cluster algebras are associated with polytopes generalizing the associahedron [25], one can conjecture that in kinematic space $\left(\mathrm{dS}_{2} \times \mathrm{dS}_{2}\right)$ the new patterns of entanglement are represented in a mathematically natural manner. In particular, in the static case these patterns probably show up as certain domains in $\mathrm{dS}_{2}$ (as an associated spacetime connected to such generalized polytopes) with their random walks adjoined to them. The corresponding time evolution in $\mathbb{K}$ then would dualize to some sort of evolution of entanglement in the boundary CFT. We hope that the associated order structures might provide some new insight to issues concerning quantum circuits related to holographic codes $[11,30,31]$.

\section{ACKNOWLEDGMENTS}

This work was supported by the National Research Development and Innovation Office of Hungary within the Quantum Technology National Excellence Program (Project No. 2017-1.2.1-NKP-2017-0001).

Note added.-Recently, we have become aware of a recent paper of Arkani-Hamed et al. on the topic of scattering amplitudes in the biadjoint $\phi^{3}$ theory [54], a research direction we commented in our outlook. The authors observe that the physical origin for the occurrence of polytopes like the associahedron is associated with causal structures in a space they call "kinematic spacetime" similar to our $\mathbb{K}$ used here. Moreover, they discover the very same structures (cluster algebras, wave equation, causal structures, and walk) in their scattering context. Now the challenge is to understand these results in terms of the entanglement picture presented here. Hopefully, it will also offer a new twist to the idea of holography.
[1] S. Ryu and T. Takayanagi, Phys. Rev. Lett. 96, 181602 (2006).

[2] S. Ryu and T. Takayanagi, J. High Energy Phys. 08 (2006) 045.

[3] V. E. Hubeny, M. Rangamani, and T. Takayanagi, J. High Energy Phys. 07 (2007) 062.

[4] N. Lashkari, M. B. McDermott, and M. Van Raamsdonk, J. High Energy Phys. 04 (2014) 195.

[5] T. Faulkner, M. Guica, T. Hartman, R. C. Myers, and M. van Raamsdonk, J. High Energy Phys. 03 (2014) 051.
[6] M. van Raamsdonk, Gen. Relativ. Gravit. 42, 2323 (2010).

[7] B. Czech, L. Lamprou, S. McCandlish, and J. Sully, J. High Energy Phys. 10 (2015) 175.

[8] B. Czech, L. Lamprou, S. McCandlish, B. Mosk, and J. Sully, J. High Energy Phys. 07 (2016) 129.

[9] J. de Boer, M. P. Heller, R. C. Myers, and Y. Neiman, Phys. Rev. Lett. 116, 061602 (2016).

[10] B. Czech, L. Lamprou, S. McCandlish, and J. Sully, Phys. Rev. Lett. 120, 091601 (2018). 
[11] B. Czech, L. Lamprou, S. McCandlish, and J. Sully, J. High Energy Phys. 07 (2016) 100.

[12] C. T. Asplund, N. Callebaut, and C. Zukowski, J. High Energy Phys. 09 (2016) 154.

[13] R. F. Penna and C. Zukowski, J. High Energy Phys. 07 (2019) 045.

[14] C. T. Asplund, N. Callebaut, and C. Zukowski, J. High Energy Phys. 09 (2016) 154.

[15] N. Callebaut, J. High Energy Phys. 02 (2019) 153.

[16] J. deBoer, F. M. Haehl, M. P. Heller, and R. C. Myers, J. High Energy Phys. 08 (2016) 162.

[17] P. Lévay, Phys. Rev. D 100, 126022 (2019).

[18] R. C. Penner, Commun. Math. Phys. 113, 299 (1987).

[19] R. C. Penner, Decorated Teichmüller Theory, The QGM Master Class Series Vol. 1 (European Mathematical Society, 2012), p. 377.

[20] M. A. Nielsen and I. L. Chuang, Quantum Computation and Quantum Information (Cambridge University Press, Cambridge, England, 2000).

[21] H. Casini and M. Huerta, Phys. Lett. B 600, 142 (2004).

[22] J. Zhang and B. Chen, J. High Energy Phys. 01 (2017) 092.

[23] S. Fomin and A. Zelevinsky, J. Am. Math. Soc. 15, 497 (2002).

[24] L. K. Williams, Bull. Am. Math. Soc. 51, 1 (2014).

[25] S. Fomin, M. Shapiro, and D. Thurston, Acta Math. 201, 83 (2008).

[26] A. B. Zamolodchikov, Phys. Lett. B 253, 391 (1991).

[27] E. Frenkel and A. Szenes, Math. Res. Lett. 2, 677 (1995).

[28] F. Ravanini, A. Valleriani, and R. Tateo, Int. J. Mod. Phys. A 08, 1707 (1993).

[29] F. Gliozzi and R. Tateo, Phys. Lett. B 348, 84 (1995).

[30] T. J. Osborne and D. E. Stiegeman, arXiv:1706.08823.

[31] F. Pastawski, B. Yoshida, D. Harlow, and J. Preskill, J. High Energy Phys. 06 (2015) 149.

[32] N. Arkani-Hamed, Y. Bai, S. He, and G. Yan, J. High Energy Phys. 05 (2018) 096.

[33] D. Tamari, Nieuw Arch. Wisk. 10, 131 (1962).
[34] N. Balazs and A. Voros, Phys. Rep. 143, 109 (1986).

[35] H. Maxfield, J. High Energy Phys. 04 (2015) 031.

[36] J. D. Brown and M. Henneaux, Commun. Math. Phys. 104, 207 (1986).

[37] G. Lamé, J. Math. Pures Appl. 3, 507 (1838).

[38] J. D. Stasheff, Trans. Am. Math. Soc. 108, 275 (1963).

[39] S. Huang and D. Tamari, J. Comb. Theory Ser. A 13, 7 (1972).

[40] M. K. Bennett and G. Birkhoff, Algebr. Univ. 32, 115 (1994).

[41] W. Geyer, Discrete Math. 133, 99 (1994).

[42] L. D. Faddeev and A. Y. Volkov, in Discrete Integrable Geometry and Physics (Clarendon Press, Oxford, 1999), p. 301.

[43] L. D. Faddeev and A. Y. Volkov, J. Phys. A 41, 194008 (2008).

[44] R. M. Kashaev, arXiv:0810.4352.

[45] R. Jackiw, Nucl. Phys. B252, 343 (1985).

[46] C. Teitelboim, Phys. Lett. 126B, 41 (1983).

[47] N. Callebaut and H. Verlinde, J. High Energy Phys. 05 (2019) 045.

[48] F. Gliozzi and R. Tateo, Int. J. Mod. Phys. A 11, 4051 (1996).

[49] J. Danciger, J. Topol. 7, 1118 (2014).

[50] D. Brill, in Black Holes and Wormholes in 2+1 Dimensions, edited by S. Cotsakis and G. W. Gibbons, Mathematical and Quantum Aspects of Relativity and Cosmology. Lecture Notes in Physics Vol. 537 (Springer, Berlin, 2000), p. 143.

[51] K. Skenderis and B. C. van Rees, Commun. Math. Phys. 301, 583 (2011).

[52] S. Ammineborg, I. Bengtsson, D. Brill, S. Holst, and P. Peldan, Classical Quantum Gravity 15, 627 (1998).

[53] M. Banados, C. Teitelboim, and J. Zanelli, Phys. Rev. Lett. 69, 1849 (1992).

[54] N. Arkani-Hamed, S. He, G. Salvatori, and H. Thomas, arXiv:1912.12948. 